\title{
Models and measurements of energy-dependent quenching
}

\author{
Julia Zaks · Kapil Amarnath • \\ Emily J. Sylak-Glassman • Graham R. Fleming
}

Received: 18 March 2013/Accepted: 21 May 2013/Published online: 23 June 2013

(c) The Author(s) 2013. This article is published with open access at Springerlink.com

\begin{abstract}
Energy-dependent quenching (qE) in photosystem II (PSII) is a pH-dependent response that enables plants to regulate light harvesting in response to rapid fluctuations in light intensity. In this review, we aim to provide a physical picture for understanding the interplay between the triggering of $\mathrm{qE}$ by a $\mathrm{pH}$ gradient across the thylakoid membrane and subsequent changes in PSII. We discuss how these changes alter the energy transfer network of chlorophyll in the grana membrane and allow it to switch between an unquenched and quenched state. Within this conceptual framework, we describe the biochemical and spectroscopic measurements and models that have been used to understand the mechanism of $\mathrm{qE}$ in plants with a focus on measurements of samples that perform $\mathrm{qE}$ in response to light. In addition, we address the outstanding questions and challenges in the field. One of the current challenges in gaining a full understanding of $\mathrm{qE}$ is the difficulty in simultaneously measuring both the photophysical mechanism of quenching and the physiological state of the thylakoid membrane. We suggest that new
\end{abstract}

Julia Zaks, Kapil Amarnath, Emily J. Sylak-Glassman have contributed equally.

J. Zaks · K. Amarnath · E. J. Sylak-Glassman ·

G. R. Fleming $(\square)$

Physical Biosciences Division, Lawrence Berkeley National Laboratory, 1 Cyclotron Road, Berkeley, CA 94720, USA

e-mail: grfleming@lbl.gov

J. Zaks · G. R. Fleming

Graduate Group in Applied Science and Technology, University of California, Berkeley, CA 94720, USA

K. Amarnath · E. J. Sylak-Glassman · G. R. Fleming Department of Chemistry, University of California at Berkeley, Berkeley, CA 94720, USA experimental and modeling efforts that can monitor the many processes that occur on multiple timescales and length scales will be important for elucidating the quantitative details of the mechanism of $\mathrm{qE}$.

Keywords Non-photochemical quenching . Energy-dependent quenching - Fluorescence yield . Fluorescence lifetime

\section{Introduction}

Oxygen-evolving photosynthetic organisms regulate light harvesting in photosystem II (PSII) in response to rapid changes in light intensity which occur during intermittent shading (Kulheim et al. 2002). Plants can, within seconds to minutes, turn on or off mechanisms that dissipate excess energy. The speed of these changes is faster than can be accounted for by changing gene expression, which can only take place within tens of minutes (Eberhard et al. 2008). From an engineering standpoint, the ability of a plant to dynamically regulate the behavior of the membrane without modifying its protein composition is particularly impressive. The design principles of this regulation would be useful as a blueprint for artificial photosynthetic systems such as solar cells and for engineering plants to optimize biomass or production of a natural product.

Energy is absorbed by chlorophyll in antenna proteins, which are transmembrane pigment-protein complexes in the thylakoid membrane (Blankenship 2002). The absorbed energy is then transferred to PSI and -II reaction centers (RCs) in the thylakoid membrane which convert the excitation energy to chemical energy through a charge separation event. Charge separation begins a chain of electron transport reactions that ultimately lead to the reduction of 
$\mathrm{NADP}^{+}$to NADPH and to the production of ATP. When the rate of energy absorption exceeds the rate at which energy can be used in the electron transport chain, excited chlorophyll can no longer be efficiently quenched at the RC. The accumulation of excited chlorophyll $\left({ }^{1} \mathrm{Chl}{ }^{*}\right)$ in PSII is dangerous to the plant. One major damage pathway is oxidative damage, which can occur when unquenched $\left({ }^{1} \mathrm{Chl} *\right)$ undergoes intersystem crossing (ISC) to form triplet-state chlorophyll $\left({ }^{3} \mathrm{Chl}\right.$ ) (Durrant et al. 1990). ${ }^{3} \mathrm{Chl}$ reacts with ground state oxygen to generate ${ }^{1} \mathrm{O}_{2}$, which can damage PSII (Barber 1994; Melis 1999). To reduce oxidative damage, plants have evolved mechanisms through which they are able to dissipate excess energy harmlessly. These mechanisms are collectively called non-photochemical quenching (NPQ) because the quenching does not result in the productive storage of energy.

There are NPQ mechanisms in all oxygen-evolving photosynthetic organisms, including cyanobacteria, algae, mosses, and plants (Niyogi and Truong 2013). Most of the work studying NPQ mechanisms has been done in plants. The mechanisms of NPQ in plants are generally broken down into energy-dependent quenching $(\mathrm{qE})$, state transitions (qT) (Minagawa 2011), photoinhibition quenching (qI) (Müller et al. 2001), and zeaxanthin-dependent quenching (qZ) (Nilkens et al. 2010). Mechanisms are sometimes grouped by the timescales of activation and relaxation (Demmig-Adams and Winter 1988). Because the processes that give rise to NPQ are not fully understood, it is not clear whether the different components of NPQ involve entirely different mechanisms.

Efforts to understand qE have been underway for over 45 years, primarily on plants, but the mechanisms associated with qE are not fully known. In Fig. 1, we propose a definition of what it would mean to fully understand $\mathrm{qE}$, inspired by Fig. 2 from Ruban's 2012 review (Ruban et al. 2012). Firstly, it is necessary to understand the trigger or what conditions cause $\mathrm{qE}$ to turn on. While it is known that a $\mathrm{pH}$ gradient $(\Delta \mathrm{pH})$ across the thylakoid membrane triggers $\mathrm{qE}$ (Ruban et al. 2012), to fully understand the role of the $\mathrm{pH}$ trigger, it is necessary to characterize the modifications of $\mathrm{pH}$-sensitive moieties. Secondly, it is important to understand the membrane changes that occur to create a qE-active state and how the properties of particular pigments are altered to be able to rapidly quench excitation. It is thought that a macroscopic membrane rearrangement may induce conformational changes in individual proteins that affect the interactions between pigments, changing the energy transfer dynamics (Betterle et al. 2009; Johnson and Ruban 2011). Lastly, it is crucial to understand the photophysical quenching mechanisms, where and how quenching occurs. The mechanism and the location of quenching have been under debate for many years.
Quenching through chlorophyll-chlorophyll interactions (Beddard and Porter 1976; Miloslavina et al. 2008; Müller et al. 2010) and chlorophyll-carotenoid interactions (Ahn et al. 2008; Bode et al. 2009; Gilmore et al. 1995; Holt et al. 2005; Pascal et al. 2005; Ruban et al. 2007) have been proposed. qE has been studied by researchers from a broad range of fields. This diversity of approaches has led to a wide variety of theoretical and experimental tools that have been valuable in studying qE.

In this paper, we review the methods and techniques that have been used in $\mathrm{qE}$ research. These methods, though often developed and primarily used to study plants, can be used to study $\mathrm{qE}$ in any photosynthetic organism, and many can be used to study any NPQ mechanism. We focus on the applications of these methods to samples that are capable of performing $\mathrm{qE}$ in response to light, such as thylakoids, chloroplasts, and whole leaves, and do not review many experiments done on isolated and aggregated proteins. For a review of experiments on isolated complexes, see Ruban et al. (2012). We also limit the scope of this review to the application of these methods to $\mathrm{qE}$ in plants, although other organisms, such as cyanobacteria, also exhibit NPQ processes that have similarities with qE. Some methods, such as the use of fluorescence yield measurements, chemical inhibitors, and $\mathrm{qE}$ mutants, have been used to extract information about all parts of the $\mathrm{qE}$ process: the trigger, membrane change, and photophysical mechanism of quenching. We discuss the use of these methods, as well as their strengths and limitations, in the "General tools for the study of $\mathrm{qE}$ " section. In the "Triggering of $\mathrm{qE}$ " section, we discuss the current understanding of the trigger by reviewing methods and models for correlating $\mathrm{qE}$ with the lumen $\mathrm{pH}$. We discuss the techniques used to monitor membrane changes and to identify the quenching site(s) and photophysical mechanism(s) of NPQ in the "Formation of $\mathrm{qE}$ in the grana membrane" section. Finally, in the "New tools for characterizing $\mathrm{qE}$ in vivo" section, we discuss the development of measurements and techniques to study the dynamics of $\mathrm{qE}$ in vivo.

\section{General tools for the study of $\mathrm{qE}$}

\section{Discovery and early studies of $\mathrm{qE}$}

$\mathrm{qE}$ was first observed in fluorescence studies of isolated chloroplasts subjected to chemical treatments. The amount of chlorophyll fluorescence was found to depend on the $\mathrm{pH}$ of the lumen. Figure 3 illustrates the series of experiments performed by Murata and Sugahara (1969) and Wraight and Crofts (1970). Chloroplasts were first treated with dichlorophenyl-dimethylurea (DCMU), which inhibits 
Fig. 1 To understand the mechanism of $\mathrm{qE}$ requires an understanding of the dynamics of the trigger, the membrane change, and the photophysical mechanism. The techniques that are used to study the different aspects of the mechanism are listed below the respective process

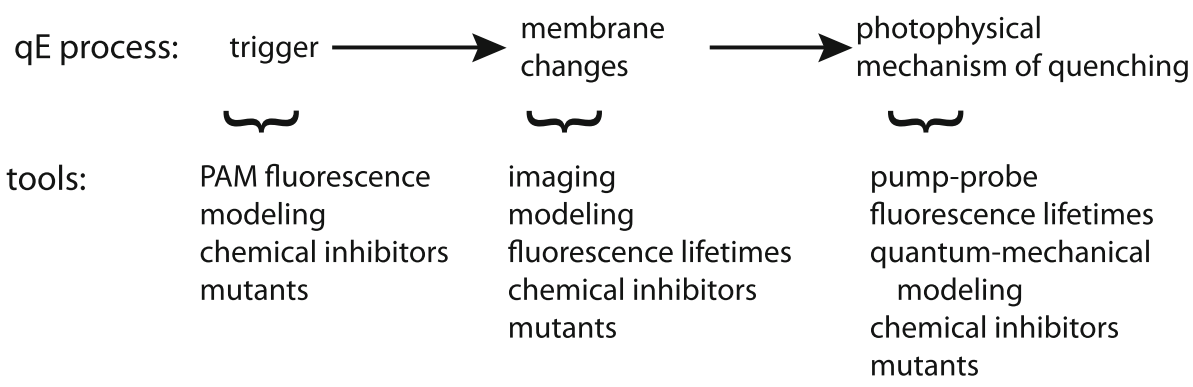

electron transfer at PSII and prevents photochemical quenching. Because excited chlorophyll could not be quenched photochemically (by charge separation at the $\mathrm{RC}$ ), a high level of fluorescence was observed. The addition of mediators of cyclic electron flow (either phenazine methosulfate [PMS] Murata and Sugahara 1969 or diaminodurene [DAD] Wraight and Crofts 1970), which stimulate the formation of a $\Delta \mathrm{pH}$ across the thylakoid membrane, quenched the fluorescence. This quenching was eliminated by the addition of ionophores that dissipated the $\Delta \mathrm{pH}$, but was not eliminated by dissipation of the electric field gradient $\Delta \psi$. These experiments led to the observation that this "energy-dependent quenching," now abbreviated as $\mathrm{qE}$, is triggered by the $\Delta \mathrm{pH}$ across the thylakoid membrane. Nearly a decade after these initial studies of a $\mathrm{pH}$ dependent quenching mechanism, Briantais et al. (1979) found that this phenomenon was not something that could only be seen under artificial treatments, but occurs naturally when plants are illuminated. Briantais and coworkers correlated the chlorophyll fluorescence with the $\mathrm{pH}$ of the lumen by measuring the $\mathrm{pH}$-dependent fluorescence of 9-aminoacridine. They found that illuminated chloroplasts' fluorescence yield decreases as the $\mathrm{pH}$ decreases. This result indicated that $\mathrm{qE}$ occurs naturally and not just with chemical treatments. The use of chemicals to block linear electron transport and uncouple the $\mathrm{pH}$ and electric field gradients is still a useful technique for studying $\mathrm{qE}$.

Fluorescence yield measurements

Chlorophyll fluorescence yield is the most frequently used quantity for observing $\mathrm{qE}$. Because the chlorophyll fluorescence yield depends on the rates of relaxation for excited state chlorophyll, it can be used to determine the amount of photochemical quenching and NPQ (Krause and Weis 1991). Additionally, the fluorescence yield can be detected non-invasively, which has allowed researchers to measure the fluorescence yield in living photosynthetic organisms such as green algae and leaves as they respond to changing light conditions both in the laboratory and in the field.

Early fluorescence measurements (Murata and Sugahara 1969; Wraight and Crofts 1970) detected the absolute

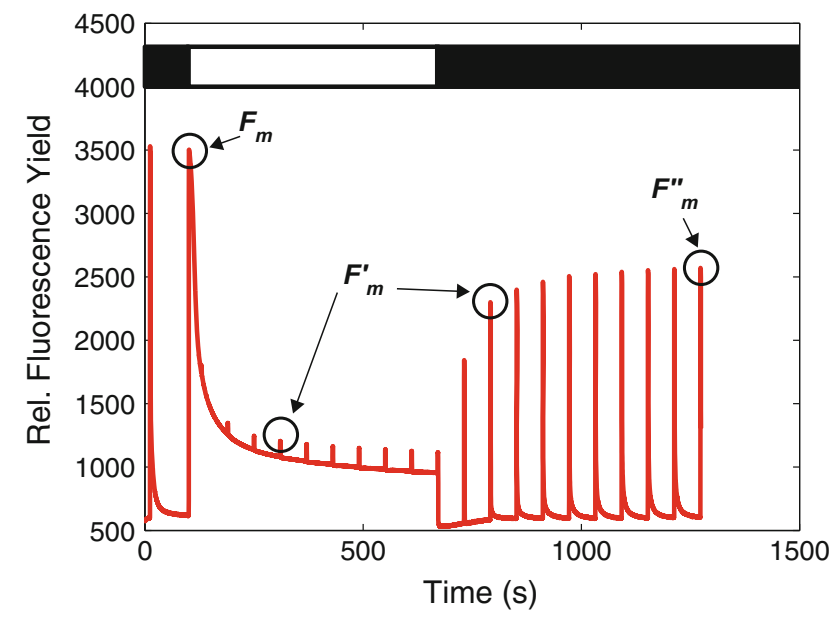

Fig. 2 A PAM trace of a leaf from Arabidopsis thaliana is shown in red. The bar at the top of the figure indicates periods of darkness (black) and actinic light illumination at an intensity of $680 \mu \mathrm{mol}$ photons $\mathrm{m}^{-2} \mathrm{~s}^{-1}$ (white). The saturating pulses occurred wherever there is a spike in fluorescence. The trace was averaged over six different leaves. The $F_{\mathrm{m}}$ peak and the $F_{\mathrm{m}}^{\prime \prime}$ peaks are indicated. The $F_{\mathrm{m}}^{\prime}$ peaks are all the peaks in fluorescence that are not $F_{\mathrm{m}}$ and $F_{\mathrm{m}}^{\prime \prime}$, and only two of them are pointed out for clarity

fluorescence from an illuminated sample and how it changed following different chemical treatments. Because the total fluorescence is proportional to the illumination intensity, comparing the amount of fluorescence across different illumination conditions requires measuring of the fluorescence quantum yield, $\phi_{\mathrm{F}}$.

$\phi_{\mathrm{F}}=\frac{\text { number of photons emitted }}{\text { number of photons absorbed }}$.

PAM fluorimetry is a widely used tool for measuring changes in the chlorophyll fluorescence yield as plants acclimate to changing light conditions (Schreiber et al. 1986). PAM techniques are reviewed in Brooks and Niyogi (2011) and Schreiber (2004). While absolute fluorescence measurements use a single light source to illuminate the sample and induce fluorescence, PAM fluorimeters only detect fluorescence resulting from a low intensity $\left(<0.1 \mu\right.$ mol photons $\left.\mathrm{m}^{-2} \mathrm{~s}^{-1}\right)$ modulated measuring light that minimally affects the photochemistry or NPQ in the plant. 


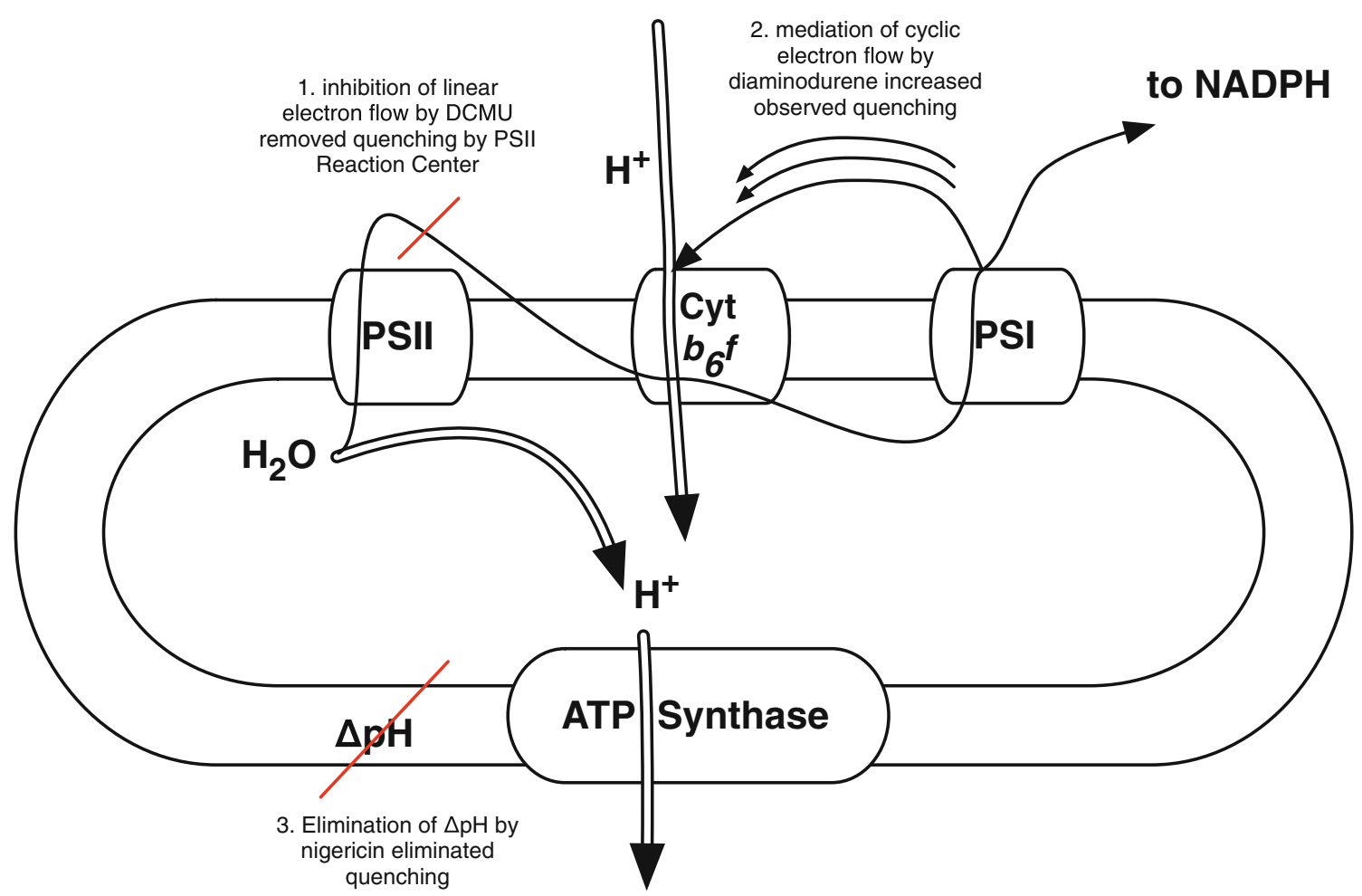

Fig. 3 Schematic of experiment performed by Wraight and Crofts (1970) to identify that the $\Delta \mathrm{pH}$ was the trigger for $\mathrm{qE}$. The thin black arrows indicate electron flow and the thick arrows with the white stems refer to proton movement. In the experiment, chloroplasts were treated with DCMU to prevent quenching by the PSII reaction center. The addition of diaminodurene to these chloroplasts lowered the

Typical qE PAM fluorimeter measurements consist of a dark-acclimated sample exposed to actinic light (light that results in productive photosynthesis) until $\mathrm{qE}$ reaches a steady state (approximately $10 \mathrm{~min}$ ), followed by a period of dark reacclimation until $\mathrm{qE}$ turns off. To distinguish the effects of photochemical quenching (irreversible charge separation in the RC) and NPQ, fluorescence yield measurements are compared when PSII RCs are open and closed. RCs are considered to be open when the primary plastoquinone electron acceptor in the RC, $Q_{\mathrm{A}}$, is oxidized and is considered closed when $Q_{\mathrm{A}}$ is reduced (Baker 2008; Govindjee 2004). During the illumination and dark periods, short $(<1 \mathrm{~s}$ ) pulses of high intensity (up to $20,000 \mu \mathrm{mol}$ photons $\mathrm{m}^{-2} \mathrm{~s}^{-1}$ ) actinic light are used to close PSII RCs. When RCs are open, excited chlorophyll can relax via photochemical quenching, NPQ, fluorescence, or ISC. When the saturating pulses close the RCs, the only available pathways are NPQ, fluorescence, or ISC. The rates of these processes affect the measured fluorescence quantum yield.

To characterize the NPQ response of a plant, it is useful to compare the fluorescence yield when the PSII RCs are closed before and during light acclimation. $F_{\mathrm{m}}$ is proportional to the maximum fluorescence yield measured during lumen $\mathrm{pH}$ via cyclic electron flow and caused chlorophyll fluorescence to be quenched. This quenching was eliminated by the addition of nigericin and dianemycin, which dissipate the $\mathrm{pH}$ gradient. The quenching was much less sensitive to the addition of valinomycin, which dissipates the electric field across the membrane

a saturating pulse of actinic light applied to dark-acclimated leaves. $F_{\mathrm{m}}^{\prime}$ is the maximum fluorescence yield following exposure to light, also measured during saturating pulses. A parameter called NPQ can be calculated with these parameters (Schreiber et al. 1994).

$\mathrm{NPQ}=\frac{F_{\mathrm{m}}-F_{\mathrm{m}}^{\prime}}{F_{\mathrm{m}}^{\prime}}$.

This NPQ parameter is useful to compare relative amounts of quenching between various mutants and light conditions because it increases as quenching turns on and decreases as quenching turns off. The derivation and use of this NPQ parameter are described in greater detail in the Appendix A and in Ahn et al.(2009), Baker (2008), Brooks and Niyogi (2011), and Holzwarth et al. (2013). To separate qE from qT, $\mathrm{qZ}$, and $\mathrm{qI}, F_{\mathrm{m}}^{\prime \prime}$, the maximum fluorescence yield after qE has relaxed, is often measured (Ahn et al. 2009; Johnson and Ruban 2011) and used instead of $F_{\mathrm{m}}^{\prime}$ in Eq. 2.

PAM traces also allow researchers to quickly assay the $\mathrm{qE}$ response with different mutants, light conditions, and chemical treatments. These measurements are often correlated with biochemical measurements that quantify parameters such as the protein or pigment content (for 
example, Betterle et al. 2009; Nilkens et al. 2010; Niyogi et al. 1998) to investigate the relationship between these components and $\mathrm{qE}$.

\section{Chemical inhibitors}

Chemical inhibitors have been used in in vitro measurements to perturb a plant's $\mathrm{qE}$ response, often by inhibiting particular steps of photosynthetic electron transport (see Table 1). DCMU is commonly used to close RCs (Murata and Sugahara 1969) by blocking the electron flow from PSII to plastoquinone pool, effectively closing the RCs without using saturating light, as is done in PAM fluorimetry (Clayton et al. 1972). In this way, DCMU allows researchers to take measurements without photochemical quenching present. This allows for the isolation of NPQ processes without the complications of photochemical processes.

Ionophores are used in $\mathrm{qE}$ studies to alter the $\Delta \mathrm{pH}$ and/ or $\Delta \psi$. Nigericin is a commonly used chemical inhibitor in $\mathrm{qE}$ studies (Heldt et al. 1973). Nigericin is a protonpotassium anti-porter that dissipates the $\Delta \mathrm{pH}$ across the thylakoid membrane and eliminates qE. In both in vitro and in vivo studies, it has been particularly useful because it can be added while $\mathrm{qE}$ is already activated to dissipate the $\Delta \mathrm{pH}$ (Amarnath et al. 2012; Johnson and Ruban 2010). The addition of nigericin separates $\mathrm{qE}$ from the other NPQ components. There are other chemicals that can be used to alter the electrochemical gradient. Gramicidin and carbonylcyanide $m$-chlorophenylhydrazone (CCCP) dissipate both $\Delta \mathrm{pH}$ and $\Delta \psi$ (Nishio and Whitmarsh 1993). Valinomycin, a potassium transporter, dissipates only the $\Delta \psi$
(Wraight and Crofts 1970). These treatments were used to determine that the $\Delta \mathrm{pH}$, not the $\Delta \psi$, is the trigger for $\mathrm{qE}$, as described in the introduction of this Section.

$N, N^{\prime}$-dicyclohexylcarbodiimide (DCCD) binds to protonatable carboxylate groups accessible to the lumen in the hydrophobic region of proteins (Ruban et al. 1992). It has been used to determine whether a protein is $\mathrm{pH}$ sensitive and to identify protonatable residues in antenna complexes of PSII (Walters et al. 1996) and the protein PsbS (Dominici et al. 2002; Li et al. 2002b).

The enhancement of cyclic electron flow around PSI by chemical electron donors and acceptors such as PMS and DAD led to the discovery of $\mathrm{qE}$, as discussed in the introduction of this section. This approach has been used to provide information about the trigger of $\mathrm{qE}$ because it enables researchers to manipulate the $\mathrm{pH}$ of the lumen without involving PSII. As an example, DAD has been used to decrease the $\mathrm{pH}$ of the lumen below physiological levels to investigate $\mathrm{qE}$ in mutants of Arabidopsis thaliana (Johnson and Ruban 2011).

More generally, a challenge in using chemical inhibitors is that they may have multiple interactions in the chloroplast that are not fully known or characterized. As a result, pathways other than the desired one may be affected.

$\mathrm{qE}$ mutants

Plant mutants that display enhanced or inhibited quenching have aided in identifying the components that are necessary to see a full $\mathrm{qE}$ response. Many of these mutants were created by randomly mutating $A$. thaliana seeds by fast neutron bombardment, treatment with ethylmethyl sulfinate
Table 1 Chemical treatments used to study qE

\begin{tabular}{|c|c|}
\hline Names & Effects \\
\hline$N, N^{\prime}$-dicyclohexylcarbodiimide (DCCD) & $\begin{array}{l}\text { Binds to protonatable protein carboxylate groups } \\
\text { (Ruban et al. 1992) }\end{array}$ \\
\hline $\begin{array}{l}\text { 3-(3,4-Dichlorophenyl)-1,1-dimethylurea } \\
\text { (DCMU) }\end{array}$ & $\begin{array}{l}\text { Blocks electron flow from PSII to plastoquinone, closes } \\
\text { PSII reaction centers (Murata and Sugahara 1969) }\end{array}$ \\
\hline Nigericin & Eliminates $\Delta \mathrm{pH}$ (Heldt et al. 1973) \\
\hline $\begin{array}{l}\text { Carbonylcyanide } m \text {-chlorophenylhydrazone } \\
\text { (DCCP) }\end{array}$ & Dissipates $\Delta \mathrm{pH}$ and $\Delta \Psi$ (Nishio and Whitmarsh 1993) \\
\hline Dithiothreitol (DTT) & $\begin{array}{l}\text { Inhibits violaxanthin de-epoxidase (Yamamoto and } \\
\text { Kamite 1972) }\end{array}$ \\
\hline Gramicidin & Eliminates $\Delta \mathrm{pH}$ and $\Delta \Psi$ (Nishio and Whitmarsh 1993) \\
\hline Dibromothymoquinone (DBMIB) & $\begin{array}{l}\text { Blocks electron flow from plastoquinone to cytochrome } \\
b_{6} f \text { (Nishio and Whitmarsh 1993) }\end{array}$ \\
\hline Methyl viologen & Electron acceptor (Nishio and Whitmarsh 1993) \\
\hline Diaminodurene (DAD) & $\begin{array}{l}\text { Mediator of cyclic electron flow (Wraight and Crofts } \\
\text { 1970) }\end{array}$ \\
\hline Phenazine methosulfate (PMS) & $\begin{array}{l}\text { Mediator of cyclic electron flow (Murata and Sugahara } \\
\text { 1969) }\end{array}$ \\
\hline Valinomycin & Eliminates $\Delta \Psi$ (Wraight and Crofts 1970) \\
\hline
\end{tabular}


(EMS), or transfer DNA. Seedlings are selected and characterized by their fluorescence yield, often using a video imaging technique developed by Niyogi et al. (1998) that allows for rapid visualization of NPQ on a large number of mutagenized seedlings. Plants with altered NPQ levels compared to wild type can then be further characterized. This method allowed for the identification of many $\mathrm{qE}$ mutants. These mutants are listed in Table 2.

Arabidopsis thaliana mutants have provided researchers with a method of removing or altering proteins in the photosynthetic apparatus. Examples include the mutants which showed that the protein PsbS is necessary for $\mathrm{qE}$. In wild type plants grown in low light, there are approximately 2 PsbS per PSII (Funk et al. 1995). The $n p q 4$ mutant, which lacks PsbS, shows no qE in PAM traces, demonstrating that PsbS is necessary for $\mathrm{qE}$ in vivo $(\mathrm{Li}$ et al. 2000). The $n p q 4-E 122 Q$ and $n p q 4-E 226 Q$ mutants, each of which has one lumen-exposed glutamate residue mutated such that it cannot be protonated, have $\mathrm{qE}$ levels that are midway between that of the wild type and $n p q 4$. This showed that PsbS is $\mathrm{pH}$ sensitive and likely undergoes some conformational change when the lumen $\mathrm{pH}$ is low $(\mathrm{Li}$ et al. 2002b). To further examine the role of PsbS, the npq4-1 mutant was complemented with the wild type PsbS gene, yielding a set of mutants with varying levels of PsbS (Niyogi et al. 2005). The qE levels of these mutants show that the maximum $\mathrm{qE}$ level increases with increasing ratio of PsbS to PSII (Niyogi et al. 2005). This increase eventually plateaus when the level of PsbS is 6-8 times that of the wild type. Additionally, two mutants that contain elevated levels of PsbS, L5 and L17, exhibit approximately twice the amount of NPQ compared to wild type plants.
These mutants have revealed that the capacity for $\mathrm{qE}$ in wild type A. thaliana is not saturated and can be increased by elevating PsbS levels.

Because of the complexity and interconnectedness of the thylakoid membrane, removing one component, such as a pigment or a protein, may cause other components in the membrane to compensate in a manner that is challenging to predict and characterize. One example of this is the mutant npq1, which cannot convert violaxanthin to zeaxanthin (Niyogi et al. 1998). However, the mutation does not block the biosynthesis of zeaxanthin from $\beta$-carotene. Therefore, while npql has a strongly reduced amount of zeaxanthin, some zeaxanthin and antheraxanthin are still present. In the case of $n p q 2$, which lacks zeaxanthin epoxidase, zeaxanthin accumulates even in the dark, so quenching components related to $\mathrm{qZ}$ are always present in the $n p q 2$ mutant. This additional quenching is reflected in the lower $F_{\mathrm{v}} / F_{\mathrm{m}}$ quantity in $n p q 2$ that is used to characterize the plant's capacity for photochemistry and limits the qE-specific information that can be extracted from studies of the $n p q 2$ mutant.

Another caution in using mutants is that changing one gene may have unintended consequences on the greater photosynthetic apparatus. For instance, knocking out PsbS as in $n p q 4$ could change the properties of the thylakoid membrane, which affect more processes than just $\mathrm{qE}$. PsbS has been shown to affect the stacking of the grana membranes (Kiss et al. 2008) and to affect the distance between PSII centers upon illumination (Betterle et al. 2009). These changes have not been shown to be directly related to $\mathrm{qE}$, but they complicate the interpretation of the role of PsbS. As another example, the altered $\mathrm{qE}$ dynamics of the lut 2

Table 2 A. thaliana mutants used to study qE

\begin{tabular}{|c|c|c|}
\hline Names & Mutations & Effects \\
\hline$n p q 4$ (Li et al. 2000) & Lacks PsbS function & $\begin{array}{l}\text { Decreased amount of } \mathrm{qE} \text {, slower turn on } \\
\text { and off compared to wild type }\end{array}$ \\
\hline npq1 (Niyogi et al. 1998) & No violaxanthin de-epoxidase activity & $\begin{array}{l}\text { Decreased } \mathrm{qE} \text {, slower turn on and off } \\
\text { compared to wild type }\end{array}$ \\
\hline npq2 (Niyogi et al. 1998) & No zeaxanthin epoxidase activity & $\begin{array}{l}\text { Equal } \mathrm{qE} \text {, more rapid turn on, slower turn } \\
\text { off compared to wild type }\end{array}$ \\
\hline lut2 (Pogson et al. 1998) & No production of lutein & Decreased amount of $\mathrm{qE}$ \\
\hline npq1lut2 (Niyogi et al. 2001) & See above & No $\mathrm{qE}$ \\
\hline npq4npq1lut2 (Li et al. 2002a) & See above & No qE \\
\hline L5 (Li et al. 2002a) & Over-expresses PsbS & Increased amount of $\mathrm{qE}$ \\
\hline L17 (Li et al. 2002a) & Over-expresses PsbS & Increased amount of $\mathrm{qE}$ \\
\hline$n p q 4-E 122 Q$ (Li et al. 2002b) & $\begin{array}{l}\text { One of two lumen-exposed glutamate } \\
\text { residues mutated to glutamine }\end{array}$ & $50 \% \mathrm{qE}$ compared to wild type \\
\hline$n p q 4-E 226 Q(\mathrm{Li}$ et al. 2002b) & $\begin{array}{l}\text { One of two lumen-exposed glutamate } \\
\text { residues mutated to glutamine }\end{array}$ & $50 \% \mathrm{qE}$ compared to wild type \\
\hline
\end{tabular}


mutant, which lacks lutein, may be due to the misfolding of light-harvesting proteins rather than a change in the $\mathrm{qE}$ mechanism (Dall'Osto et al. 2006). Nonetheless, the $A$. thaliana $\mathrm{qE}$ mutants have provided a powerful tool for studying the components and mechanism of $\mathrm{qE}$.

\section{Triggering of $\mathrm{qE}$}

We now turn to a description of tools to study $\mathrm{qE}$ triggering. A complete understanding of the triggering of $\mathrm{qE}$ by $\Delta \mathrm{pH}$ requires characterizing the value of the lumen $\mathrm{pH}$ at which the components of $\mathrm{qE}$ are turned on. It is important to know the $\mathrm{pH}$ level at which any $\mathrm{pH}$-sensitive $\mathrm{qE}$ components are activated and whether these $\mathrm{pH}$ levels are absolute or modulated by other environmental factors. It is also important to characterize the "steepness" of the $\mathrm{pH}$ dependence of $\mathrm{qE}$. A steep $\mathrm{pH}$ dependence would correlate to a "switch" from fully on to fully off in a short $\mathrm{pH}$ range. By contrast, a shallow $\mathrm{pH}$ dependence would correspond to a "dial," where the activation level gradually changes from off to on. In addition to quantifying the response of the proteins involved in $\mathrm{qE}$ to protonation, a complete understanding of $\mathrm{qE}$ triggering requires knowing the response of PSII to the protonation of these key proteins. This response could involve conformational changes within or between proteins and is discussed in the "Formation of $\mathrm{qE}$ in the grana membrane" section.

Although work with chemical inhibitors has convincingly shown that $\mathrm{qE}$ is triggered by acidification of the lumen, quantifying the $\mathrm{qE}$ response to lumen $\mathrm{pH}$ is challenging. This challenge arises from the fact that the complexes involved in $\mathrm{qE}$ are embedded in the thylakoid membrane and that the $\mathrm{pH}$-sensitive components of these complexes are located in the lumen space. To characterize the response of $\mathrm{qE}$ to $\Delta \mathrm{pH}$, researchers have sought to measure the lumen $\mathrm{pH}$ and determine the $\mathrm{p} K_{\mathrm{a}} \mathrm{s}$ of key proteins and enzymes. These downstream responses to the $\mathrm{pH}$ trigger have been investigated by a combination of measuring the lumen $\mathrm{pH}$ and correlating it to the amount of $\mathrm{qE}$. The effect of $\Delta \mathrm{pH}$ on $\mathrm{qE}$ has been quantified by fitting the relationship between observed $\mathrm{qE}$ quenching and measured lumen $\mathrm{pH}$ to various equations, as in Takizawa et al. (2007), Johnson and Ruban (2011), and Johnson et al. (2012). Several experimental methods have been developed to measure the lumen $\mathrm{pH}$ as well as the $\Delta \mathrm{pH}$ across the thylakoid membrane. These methods rely on indirect spectroscopic measurements of lumen $\mathrm{pH}$, either by measuring fluorescence of dyes (Junge et al. 1979; Schuldiner et al. 1972) or by measuring spectroscopic signals of carotenoids (Bailleul et al. 2010; Takizawa et al. 2007). In this section, we review several recent experiments investigating the triggering of $\mathrm{qE}$.
Proteins triggered by $\Delta \mathrm{pH}$

Figure 4a illustrates the known components of $\mathrm{qE}$ in plants that respond to lumen $\mathrm{pH}$. When the $\mathrm{pH}$ of the lumen drops and $\Delta \mathrm{pH}$ is formed across the membrane, several processes in the thylakoid membrane are triggered:

(1) The enzyme violaxanthin de-epoxidase (VDE) is activated (Jahns et al. 2009). In its active form, VDE converts the carotenoid violaxanthin, which is present in several of the light-harvesting proteins of PSII, to the carotenoid zeaxanthin via the xanthophyll cycle.

(2) The protein PsbS (Funk et al. 1995), which is necessary for rapidly reversible quenching in vivo, is activated ( $\mathrm{Li}$ et al. 2000). The sensing of lumen $\mathrm{pH}$ is done by two lumen-exposed glutamates, as discussed in the "qE mutants" section.

(3) The minor light-harvesting pigment-protein complexes CPs29 and -26 contain glutamate residues that bind DCCD (Walters et al. 1996). It is possible that the protonation of these residues contributes to triggering qE. Deletion of either light-harvesting complex (LHC) from the PSII antenna (Andersson et al. 2001; Betterle et al. 2009; de Bianchi et al. 2008) does not eliminate $\mathrm{qE}$, suggesting that these complexes could play an indirect role in $\mathrm{qE}$ (Ruban et al. 2012). Nonetheless, $\mathrm{qE}$ turns on more slowly and reaches lower levels in mutants lacking CP29 (Betterle et al. 2009; de Bianchi et al. 2011).

Because the individual activation steps giving rise to $\mathrm{qE}$ cannot be measured directly, efforts to understand the relationship between lumen $\mathrm{pH}$ and the components of $\mathrm{qE}$ have largely relied on measurements of total $\mathrm{qE}$, as illustrated in Fig. 4. We review these measurements below. In general, to quantify the relationship between lumen $\mathrm{pH}$ and $\mathrm{qE}$, measurements have been fit to the Hill equation. The Hill equation can be used to describe the protonation of individual proteins, as in Fig. 4a. It is also commonly used as a more general phenomenological equation to fit data and has been directly applied to quantify the relationship between lumen $\mathrm{pH}$ and $\mathrm{qE}$, as in Fig. $4 \mathrm{~b}$.

The Hill equation has the form

$F=\frac{\left[H^{+}\right]^{n}}{\left[H^{+}\right]^{n}+\left[10^{-p K_{a}}\right]^{n}}$,

where $F$ is the fraction of proteins that are activated. The Hill equation contains two parameters: the $\mathrm{p} K_{\mathrm{a}}$, which is the $\mathrm{pH}$ at which $F=0.5$, and the Hill coefficient $n$, which is a measure of the sigmoidicity, or "steepness," of the transition of $F$ from a "100\% on" state to a "100\% off" state. In the case when a protein must bind multiple protons to be activated, and when this binding is highly 
Fig. 4 a The triggering of $\mathrm{qE}$ in plants by lumen $\mathrm{pH}$ involves the protonation of PsbS, VDE, and possibly other light-harvesting proteins. A full understanding of $\mathrm{qE}$ triggering involves quantitative knowledge of the $\mathrm{p} K_{\mathrm{a}}$ and Hill coefficient of each protonation step, as well as a characterization of the interaction between pigments and protonated proteins to form a $\mathrm{qE}$ state. b Because activation levels of individual proteins cannot be measured directly, experimental data quantifying the relationship between $\mathrm{qE}$ to lumen $\mathrm{pH}$ frequently fit the overall data phenomenologically to an effective $\mathrm{p} K_{\mathrm{a}}$ and Hill coefficient a Fitting triggering parameters of individual components

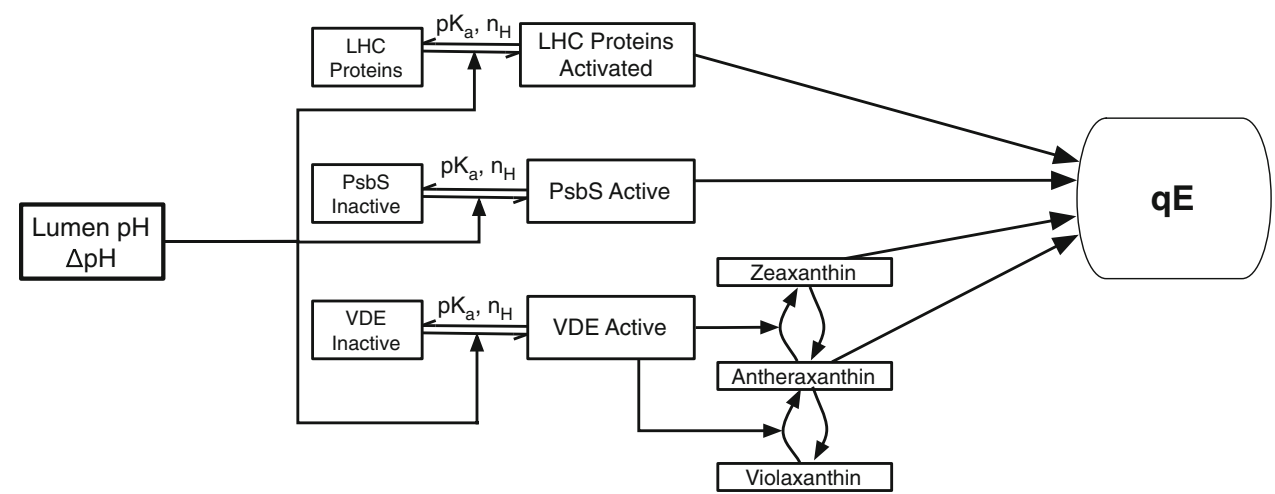

b Fitting effective parameters of overall $q E$ triggering

\begin{tabular}{|c|}
\hline Lumen $\mathrm{pH}$ \\
$\Delta \mathrm{pH}$ \\
\hline
\end{tabular}

cooperative, the Hill coefficient $n$ can be interpreted as the number of protons needed to activate the protein, as in the reaction

$A+n H^{+} \rightleftharpoons A H_{n}^{+}$.

In the case when binding is not extremely cooperative, the Hill coefficient still measures the cooperativity of binding, but does not correspond directly to a physical property such as the number of protonatable sites (Weiss 1997).

The existing measurements from several labs fit quite well to the Hill equation. However, the Hill equation does not directly correspond to a physical model in most situations (Weiss 1997). As a result, extracting mechanistic information from measurements of $\mathrm{qE}$ measured as a function of lumen $\mathrm{pH}$ is challenging. One way forward is through the development of physically motivated mathematical models that explicitly incorporate each protonation event in various hypotheses of $\mathrm{qE}$ mechanism. In the following sections, we review measurements correlating lumen $\mathrm{pH}$ and the hypotheses that have been generated from these measurements.

\section{Measurements of $\mathrm{qE}$ triggering}

\section{$\Delta p H$ or low lumen $p H ?$}

For understanding the processes triggering $\mathrm{qE}$, it is important to differentiate between those processes that only require a low lumen $\mathrm{pH}$ and processes that require a $\Delta \mathrm{pH}$ across the thylakoid membrane. The protonation of residues in PsbS, VDE, and LHC proteins can be accomplished by lowering the lumen $\mathrm{pH}$, without necessarily requiring a $\mathrm{pH}$ gradient across the thylakoid membrane. However, work by Goss et al. (2008) demonstrated that a $\mathrm{pH}$ gradient across the thylakoid membrane, along with a neutral or slightly basic stromal $\mathrm{pH}$, is required for the formation of zeaxanthin-dependent $\mathrm{qE}$. Once $\mathrm{qE}$ is formed, it is possible to maintain $\mathrm{qE}$ even in the absence of a $\mathrm{pH}$ gradient if the lumen $\mathrm{pH}$ is kept sufficiently low (Rees et al. 1992). This property was used to determine the $\mathrm{qE}$ versus pH curves in Johnson and Ruban (2011) and Johnson et al. (2012). The ability to maintain $\mathrm{qE}$ in low $\mathrm{pH}$, even without a $\Delta \mathrm{pH}$, suggests that the $\Delta \mathrm{pH}$ is required for proper insertion of zeaxanthin (Goss et al. 2008), but that other $\mathrm{pH}$-sensitive components of $\mathrm{qE}$ do not require a $\mathrm{pH}$ gradient.

\section{Correlation of $q E$ with in vivo measurements of lumen $p H$}

To quantify the response of $\mathrm{qE}$ to lumen $\mathrm{pH}$ in vivo, the Kramer group has measured the relationship between steady-state $\mathrm{qE}$ and lumen $\mathrm{pH}$. The lumen $\mathrm{pH}$ was measured spectroscopically through a measurement of the electrochromic shift (ECS), which is a signal arising from the Stark effect of the electric field across the thylakoid membrane on the energy levels of carotenoids embedded in the membrane (Bailleul et al. 2010; Witt 1979). This effect causes the absorption spectrum of carotenoids in the spectral region between 450 and $550 \mathrm{~nm}$ to shift. The extent of spectral shift is proportional to the amplitude of the electric field and as a result can be used to measure the 
transmembrane electric field. The ECS measurement can be used to probe the lumen $\mathrm{pH}$ by shuttering off the actinic light and measuring the "reverse ECS." Explanations of information that can be obtained from the ECS measurement, including measurements of the lumen $\mathrm{pH}$, are given in Bailleul et al. (2010), Cruz et al. (2001), and Takizawa et al. (2007).

To estimate the $\mathrm{p} K_{\mathrm{a}} \mathrm{s}$ of $\mathrm{PsbS}$ and of $\mathrm{qZ}$ in vivo, Takizawa and coworkers assumed that de-epoxidized xanthophyll (i.e., zeaxanthin or antheraxanthin) and protonated PsbS are the two components necessary for $\mathrm{qE}$. This assumption involved fitting to a specific mechanistic model (Fig. 4a) and excluded the possibility that the protonation of LHC proteins is a factor in $\mathrm{qE}$ activation in vivo. Nonetheless, because it followed a specific model, this assumption enabled estimates of the $\mathrm{pH}$ level at which $\mathrm{qE}$ components were activated. The $\mathrm{p} K_{\mathrm{a}}$ of PsbS activation was fitted to be 6.8 , with a Hill coefficient of $\sim 1$, and the effective $\mathrm{p} K_{\mathrm{a}}$ of $\mathrm{qZ}$ was fit to be 6.8 with a Hill coefficient of 4.3. This effort is one of the first attempts thus far to fit the activation levels of $\mathrm{qE}$ using in vivo measurements, and the results suggest that the $\mathrm{p} K_{\mathrm{a}} \mathrm{s}$ of PsbS and $\mathrm{qZ}$ are higher in vivo than the $\mathrm{p} K_{\mathrm{a}} \mathrm{s}$ for isolated glutamate ( $\mathrm{Li}$ et al. 2002b) and for VDE in vitro (Jahns et al. 2009).

Because of the challenges of estimating the lumen $\mathrm{pH}$ in vivo, the $\mathrm{p} K_{\mathrm{a}}$ values reported will surely be subject to refinement and reexamination. Nonetheless, the spectroscopic approach of estimating $\mathrm{p} K_{\mathrm{a}} \mathrm{s}$ and Hill coefficients is notable because the parameters are estimated from intact leaves. The approach of spectroscopically measuring the lumen $\mathrm{pH}$ through the ECS shift is unique and powerful in that it does not require the extraction of chloroplasts or the use of chemicals. The technique of using reverse ECS would be even more powerful it if could be extended to measure lumen $\mathrm{pH}$ over the course of light adaptation. Such a measurement could be used to fit mechanistic kinetic models of the protonation of the proteins involved in $\mathrm{qE}$. Doing so would provide a method for determining the $\mathrm{p} K_{\mathrm{a}}$ of $\mathrm{qE}$ components during the process of $\mathrm{qE}$ induction and would enable greater precision than steadystate measurements in measuring the $\mathrm{p} K_{\mathrm{a}} \mathrm{s}$ and Hill coefficients of $\mathrm{qE}$ triggering.

\section{Titration of $\mathrm{qE}$ versus lumen $\mathrm{pH}$}

Chemical treatments and mutants in A. thaliana have shown that PsbS (Li et al. 2000), zeaxanthin (DemmigAdams 1990; Niyogi et al. 1997), and lutein (Pogson et al. 1998) are responsible for the majority of $\mathrm{qE}$ in vivo. However, recent results from the Ruban group have suggested that qE-type quenching can be induced in the absence of any of these components by artificially lowering the lumen $\mathrm{pH}$ by mediating cyclic electron flow (Johnson and Ruban 2011; Johnson et al. 2012). Chloroplasts isolated from $n p q 4$ and $n p q 1$ lut 2 mutants of A. thaliana were able to quench chlorophyll fluorescence when the lumen $\mathrm{pH}$ in the chloroplasts was lowered below levels typically found in vivo. This quenching had many of the same properties of that from wild type chloroplasts, which led to the suggestion that PsbS and zeaxanthin modulate the $\mathrm{p} K$ of $\mathrm{qE}$ in the thylakoid membrane. These observations were extensions of earlier studies correlating $\mathrm{qE}$ and $\Delta \mathrm{pH}$ in wild type A. thaliana (Briantais et al. 1979).

To characterize the effect of PsbS and zeaxanthin on the $\mathrm{p} K$ of $\mathrm{qE}$, a titration of $\mathrm{qE}$ against lumen $\mathrm{pH}$ was performed (Johnson and Ruban 2011; Johnson et al. 2012). The $\Delta \mathrm{pH}$ was measured with 9-aminoacridine, and $\mathrm{qE}$ was fit to the equation

$\mathrm{qE}=\mathrm{qE}_{\max } \frac{\Delta \mathrm{pH}^{\mathrm{n}}}{\Delta \mathrm{pH}^{n}+\Delta \mathrm{pH}_{0}^{n}}$,

where $n$ is the Hill coefficient and $\Delta \mathrm{pH}_{0}(\mathrm{p} K)$ is the $\mathrm{pH}$ at which half of all protonatable residues are protonated. By assuming a stromal $\mathrm{pH}$ of 8.0, Johnson and coworkers extracted $\mathrm{p} K \mathrm{~s}$ and Hill coefficients for $\mathrm{qE}$ in the presence and absence of lutein and zeaxanthin. In this approach, the $\mathrm{p} K$ of $\mathrm{qE}$ was fit to a value of 4.2 in violaxanthin-bound $n p q 4$, and increased to a value of 6.3 in zeaxanthin-bound wild type. This approach, in which no assumptions are made about the interaction between the $\mathrm{pH}$-sensing components of $\mathrm{qE}$, is illustrated in Fig. 4b. The extracted $\mathrm{p} K$ and Hill coefficient are phenomenological parameters that serve to quantify $\mathrm{qE}$ triggering and are useful for comparing different mutants and chemical treatments. The maximum capacity for $\mathrm{qE}, \mathrm{qE}_{\max }$, was found to be $85 \%$ of the wild type value in the npq4 and lut2npql mutants. Because this capacity was relatively high, Johnson and coworkers formulated the hypothesis that the role of PsbS, zeaxanthin, and lutein is to elevate the $\mathrm{p} K$ of $\mathrm{qE}$, but that the photophysical process responsible for $\mathrm{qE}$ quenching could in principle proceed in the absence of these components at very low $\mathrm{pH}$ values. In this hypothesis, zeaxanthin and lutein have indirect roles in $\mathrm{qE}$ and are not the pigments involved in the dissipation of excitation energy (Johnson and Ruban 2011; Johnson et al. 2012; Ruban et al. 2012).

The advantage of studying $\mathrm{qE}$ in isolated chloroplasts using chemical treatments is that the chloroplast is a sufficiently intact system that $\mathrm{qE}$ can still be observed, yet the aqueous spaces within the chloroplast are accessible enough to the experimentalist that certain processes such as cyclic electron flow can be inhibited or up-regulated. Because these treatments shift the lumen $\mathrm{pH}$ far from the physiological conditions in which $\mathrm{qE}$ is normally observed, the hypotheses of $\mathrm{qE}$ mechanism formed on the basis of these studies must be subject to testing in vivo. One 
approach would be to construct quantitative predictions of hypotheses that are based on and inspired by the in vitro results and integrate those quantitative predictions into mathematical models that predict experiments such as PAM that can be non-invasively observed in a living system, as we describe in the "New tools for characterizing qE in vivo" section.

\section{Formation of $q E$ in the grana membrane}

The protonation of the $\mathrm{pH}$-sensitive proteins in the grana membrane triggers changes in PSII that turn on qE. A physical picture that captures those changes requires an understanding of how the organization of PSII and its antenna in the grana gives rise to its light-harvesting and quenching functionality (Dekker and Boekma 2005). The grana membrane is densely populated by PSII supercomplexes and major LHCIIs. LHCII is a pigment-protein complex that can reversibly bind to the exterior of PSII supercomplexes, which are composed of several pigmentprotein complexes (Fig. 5). LHCIIs are located on the periphery, and RCs are located in the interior of PSII supercomplexes. Between the LHCIIs and RCs are the aforementioned minor LHCs, CPs24, -26, and -29. Together, the LHCIIs and PSII supercomplexes form a variably

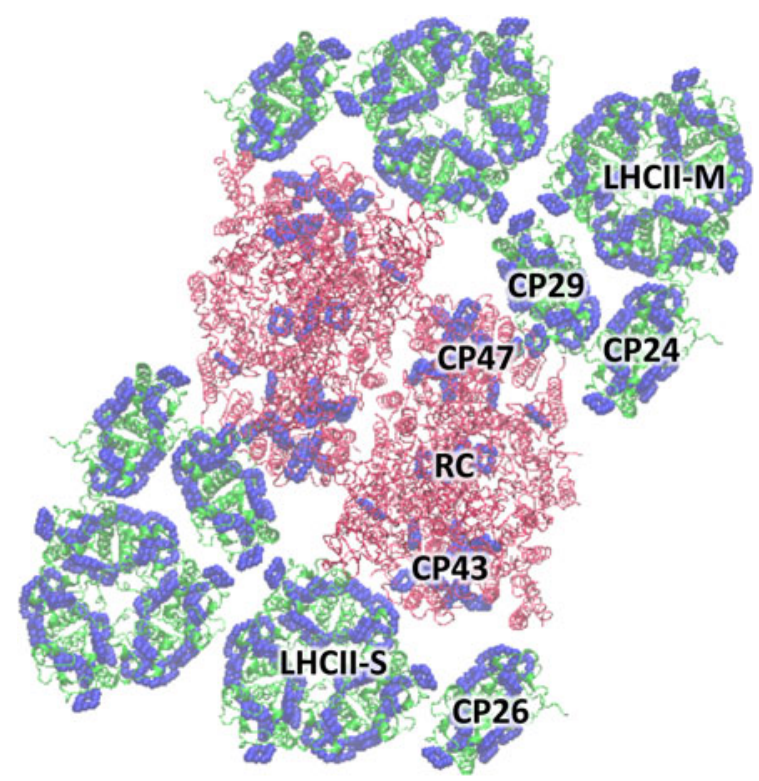

Fig. 5 Structure of the PSII supercomplex, based on the recent electron microscopy images taken by Caffarri et al. (2009). The proteins are shown as ribbons and the light-absorbing chlorin part of the chlorophyll pigments are outlined by the blue spheres. The lightharvesting antenna proteins on the exterior of the supercomplex are green, while the reaction center core (CPs47, -43, and the RC, which consists of the D1 and D2 proteins) is red. The supercomplex is a dimer. S stands for strongly bound and $\mathrm{M}$ for medium-bound LHCIIs. The supercomplex is a dimer; one of the monomers is labelled fluid array of proteins (Kouřil et al. 2012b). This array gives rise to an energy transfer network in which the pigments in the light-harvesting proteins absorb light and transfer the resulting excitation energy to RCs, where it is converted into chemical energy. In order to turn on chlorophyll quenching, this energy transfer network must change.

We represent the energy transfer network of the grana membrane using a simple grid in Fig. 6. We use this picture to illustrate the changes in the energy transfer network that may occur when $\mathrm{qE}$ turns on. It is a simplification and reduction of the complete network, which contains $\sim 100,000$ chlorophylls and the description of which has not yet been conclusively determined (Croce and van Amerongen 2011). The nodes (circles) represent groups of chlorophylls at which excitation energy can be localized and are either antenna or RCs. The dark-acclimated membrane without $\mathrm{qE}$ is shown on the left. Excitation energy can be absorbed at any nodes and transferred on the picosecond $\left(10^{-12} \mathrm{~s}\right)$ timescale along the lattice grid lines until it reaches a RC (gray nodes) (van Amerongen et al. 2000). Once it reaches a RC, the excitation energy can be "photochemically" quenched and converted into chemical energy. The $\Delta \mathrm{pH}$ triggers a series of changes in the membrane (Fig. 6, right) that change the energy transfer network on a timescale of tens of seconds to minutes. Some antennae (Havaux et al. 2007) (white nodes) gain a photophysical pathway or mechanism with a rate of relaxation to the ground state that is fast relative to fluorescence and ISC. Efficient quenching of chlorophyll excitations could prevent the excitation from reaching a RC that is susceptible to damage. To alter the properties of the pigments such that they become quenching sites may require a rearrangement of the proteins in the membrane,

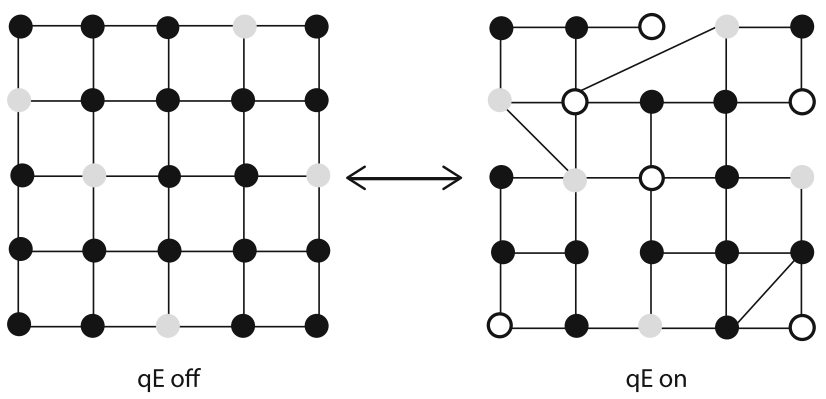

Fig. 6 A schematic of a possible configuration of chlorophyll connectivity of a portion of the grana membrane when $\mathrm{qE}$ is off (left) and when $\mathrm{qE}$ is on (right). The black circles represent nonquenching chlorophyll, such as those in LHCII. The gray circles represent PSII reaction centers, and the white circles represent $\mathrm{qE}$ quenching sites. At both reaction centers and $\mathrm{qE}$ sites, there is a rate for removing excitation from the grid. The grid lines display the connectivity for energy transfer between different groups of chlorophyll 
which is indicated by the changes in the connectivity of the network.

While this general picture of quenching is agreed on, nearly all of the details remain controversial. The energetic connectivity of pigments in the membrane is determined by their orientation, separation from other pigments, and their local protein environments. However, it is not possible at present to acquire the nearly atomic level resolution necessary for obtaining that information. Instead, a few approaches are used to study intact photosynthetic organisms. We categorize these approaches into four groups: spectroscopic measurements of pigment-pigment interactions, imaging and microscopy, fluorescence lifetimes, and transient absorption (TA) spectroscopy. Combined with modeling, these techniques can provide insight on aspects of both the membrane changes and on the site and mechanism of $\mathrm{qE}$ (Fig. 1).

Spectroscopic measurements of pigment-pigment interactions

To switch a pigment from participating in light harvesting (black node in Fig. 6) to quenching (white node) requires an alteration of its physical properties by changing its protein environment or by interactions with other pigments. Pigment-pigment interactions can be tuned by small changes in the protein conformation (van Oort et al. 2011) or by changes in the structure of a neighboring pigment, as when zeaxanthin replaces violaxanthin in high light (Crimi et al. 2001). This suggests that characterizing pigmentpigment interactions might identify pigments which are involved in $\mathrm{qE}$ and how those interactions might change to allow quenching to occur.

There are several methods to measure pigment-pigment interactions that are correlated with $\mathrm{qE}$. One spectroscopic change that occurs during $\mathrm{qE}$ is the $\Delta A_{535}$ signal (Krause 1973). This signal is determined by measuring the difference absorption spectra between light- and dark-acclimated leaves. At $535 \mathrm{~nm}$, there is an increase in absorption, which, on the basis of quantum mechanical modeling, is thought to be due to interactions between two carotenoids (violaxanthin or zeaxanthin) that occur only under $\mathrm{qE}$ conditions (Duffy et al. 2010). Another indicator of $\mathrm{qE}$ is the change in the resonance Raman spectrum of the leaf around $953 \mathrm{~cm}^{-1}$ after 5 min of exposure to actinic light (Robert 2009; Ruban et al. 2007). This change is thought to be due to changes in the conformation of a neoxanthin carotenoid in LHCII. A third indicator of $\mathrm{qE}$ is an increase in far-red fluorescence thought to be emitted from LHCII (Johnson et al. 2011; Melis 1999). The $\Delta A_{535}$ signal, the $953 \mathrm{~cm}^{-1}$ resonance Raman signal, and the fluorescence red shift have been observed in vitro under conditions that promote the aggregation of LHCII. Based partly on this evidence, Ruban and coworkers proposed that $\mathrm{qE}$ occurs due to the aggregation of LHCII in the membrane, which causes the formation of a qE quenching site (Ruban et al. 2007). Recently, the Walla group developed a method for measuring the coupling between carotenoid and chlorophyll $\mathrm{S}_{1}$ excited states and showed that this coupling increases during $\mathrm{qE}$ and correlates with qE (Bode et al. 2009; Wilk et al. 2013). Based on a proposal by van Amerongen and van Grondelle, these results were suggested to be due to an excitonic state formed between the $\mathrm{S}_{1}$ state of a carotenoid and the $Q_{\mathrm{y}}$ excited state of chlorophyll $a$ that could quickly dissipate excitation energy (Bode et al. 2009; van Amerongen et al. 2001).

\section{Imaging and microscopy}

Assessing the extent to which membrane rearrangement plays a role in $\mathrm{qE}$ requires tools that can probe the spatial arrangement of proteins in the grana membrane. Lower resolution images of the membrane that can resolve the PSIIs and LHCIIs are beneficial in determining whether a large rearrangement occurs and dramatically changes the energetic connectivity between chlorophylls. A rearrangement could be required for the conformational changes that switch a pigment into a quencher, or it could itself serve to disconnect LHCs from RCs. Protein dynamics in living systems is typically observed by tagging proteins with fluorophores. However, because most of the proteins of interest are integral membrane proteins and the grana membrane is up to $80 \%$ protein (Kirchhoff et al. 2008a), such tagging is experimentally difficult. Additionally, the tightly stacked grana membrane prevents the introduction of fluorescent proteins within the stacked region because the proteins are large compared to the space between membranes. Moreover, there are few fluorescent proteins or dyes the excitation wavelengths of which do not coincide with those of carotenoids and chlorophylls. Because the resolution limit of optical microscopy is $\sim 200 \mathrm{~nm}$, and due to the difficulties in tagging proteins of interest, protein organization in the thylakoid membrane cannot be currently resolved through confocal optical microscopy. As a result, electron microscopy (EM) and atomic force microscopy (AFM), which are more invasive than optical microscopy and can resolve features on a short length scale, have been used to image the thylakoid membrane (Dekker and Boekma 2005; Kirchhoff et al. 2008b).

EM imaging of $A$. thaliana has recently been used to understand the arrangement of proteins in the thylakoid (Boekma et al. 2000; Dekker and Boekma 2005; Kouríl et al. 2012a). Thylakoid membranes are isolated and then negatively stained for contrast. Betterle and coworkers observed that the distance between PSII centers decreased during acclimation in wild type $A$. thaliana but not in the npq4 mutant (Betterle et al. 2009). Another common EM 
technique is freeze-fracture EM, in which thylakoids are frozen and then split along the lipid bilayer such that the transmembrane proteins remain on one side of the split membrane (for review, see Staehelin 2003). Using freezefracture EM, the Ruban group observed clustering of the LHCs on the timescales of $\mathrm{qE}$ induction (Johnson et al. 2011). One drawback of using these EM techniques is the intensive sample preparation that is required. Negative staining requires fixing and dehydrating the grana, and freeze-fracture images are made with metallic replicas made from the frozen samples. In this way, the sample preparation techniques may impact the arrangement of proteins (Kirchhoff et al. 2008b). To cope with these experimental drawbacks, there has recently been effort to use cryo EM and tomography to image unstained spinach and pea chloroplasts. In cryo EM, thylakoids or chloroplasts are flash frozen at cryogenic temperatures to create vitreous samples that can then be sectioned (Dall'Osto et al. 2006; Kouřil et al. 2011). The advantage to cryo EM is that the samples remain hydrated, with the water in the sample forming a non-crystalline, vitreous ice. This technique has allowed Kouril to examine the native 3D structure of the grana membrane and the arrangement of PSII within the membrane (Kouril et al. 2012b). Although there are some experimental challenges associated with cryo EM (Daum et al. 2010; De Carlo et al. 2002), it shows much promise for future use in studying the organization of proteins in the chloroplast before and during $\mathrm{qE}$.

In addition to EM-based techniques, researchers have imaged thylakoid membranes using AFM. In AFM, samples are placed on a mica surface exposed to air and probed with a cantilever. An image is created using the height of the sample for contrast (Kirchhoff et al. 2008b). One drawback of this technique is that having the sample exposed to air rather than immersed in liquid may affect membrane properties (Fukuma et al. 2007). The application of new water-based AFM techniques (Liu et al. 2011) could probe the native rearrangements that take place in the thylakoid. Such imaging techniques should be extremely valuable for assessing the changes in chlorophyll connectivity in the membrane. In addition, thermodynamic models will be useful for understanding the strength and directionality of energetic interactions between proteins required for causing changes in membrane organization (Drepper et al. 1993; Kirchhoff et al. 2004; Schneider and Geissler 2013). It will be important to use images and models of membrane rearrangements to interpret fluorescence lifetimes, a technique that is discussed in the next section.

\section{Fluorescence lifetimes}

The chlorophyll fluorescence lifetime measures the relaxation of the chlorophyll excited state and contains information about the energy transfer network of the grana membrane. The benefits of lifetime measurements can be seen in scenarios that give rise to the same fluorescence yield, but different fluorescence lifetimes. Figure $7 \mathrm{a}$ illustrates the difference between quenching (A1), in which the lifetime of the excited state is shortened, and bleaching (A2), in which the number of fluorophores decreases. Because the fluorescence yield, which is measured in the PAM experiment, is equal to the area under the fluorescence lifetime curve, PAM measurements cannot differentiate between bleaching and quenching. Figure $7 \mathrm{~b}$ illustrates how two different energy transfer networks can be resolved by measuring fluorescence lifetimes, but not by measuring fluorescence yields.

The two decays in Fig. $7 b$ correspond to two different energy transfer networks. For instance, the fast component of B2 could be due to chlorophylls that are very close to sites with high quenching rates and the slow component due to chlorophylls far from quenching sites. The excited state lifetime is affected by any properties that affect the energy transfer network, including the location of the quenchers with respect to the light harvesters, the connectivity between chlorophylls, and the rate of quenching at $\mathrm{qE}$ sites. Extracting this information requires the use of models of energy transfer and trapping. Interpretation of fluorescence lifetime data is dependent on the sample preparation and on the energy transfer models used to analyze the data.

The methods for measuring fluorescence lifetimes include streak cameras, multi-frequency cross-correlation fluorimetry, and time-correlated single photon counting (TCSPC) (Lakowicz 2006; Noomnarm and Clegg 2009). Because TCSPC is the most commonly used method, we will focus on this technique. In TCSPC, a pulse of light excites a sample. A time $t$ later, a fluorescence photon is detected, and the arrival time is binned. After many pulses, the binned times result in a histogram that contains the excited state lifetime convolved with the instrument response function (IRF, Appendix B). The fluorescence decay is extracted by fitting exponential decay curves to the data.

A particular difficulty in performing fluorescence lifetime experiments on intact photosynthetic samples undergoing $\mathrm{qE}$ is that it takes several minutes to accumulate enough counts to obtain lifetimes that have sufficiently small confidence intervals. Gilmore et al. (1995) were able to chemically pause thylakoids undergoing qE using DTT, DCMU, and methyl viologen. Similarly, Johnson and Ruban (2009) chemically "froze" chloroplasts undergoing $\mathrm{qE}$ by the addition of protein crosslinker glutaraldehyde. The measurement of the fluorescence lifetimes of intact leaves is complicated by the fact that turning on $\mathrm{qE}$ using strong light sources instead of chemical inhibitors will induce high levels of background fluorescence or saturate the detector. To address this problem, Holzwarth et al. (2009) 


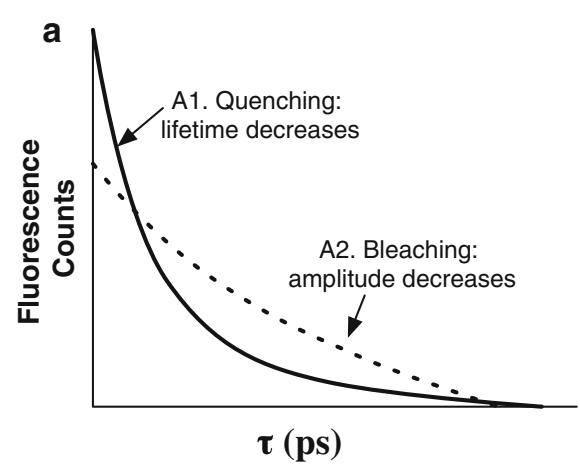

Fig. 7 Scenarios that give rise to indistinguishable fluorescence yield measurements, but that can be distinguished by fluorescence lifetime measurements. a Illustration of fluorescence lifetimes of quenching (case A1, solid line), which reduces the fluorescence lifetime, and bleaching (case A2, dashed line), which reduces the overall fluorescence amplitude. These two situations could give the same

developed a method using a rotating cuvette by which the fluorescence lifetime could be measured while $\mathrm{qE}$ was kept on.

Isolated, dilute chlorophyll has a fluorescence decay that is described by a single exponential decay with a time constant $\tau=\frac{1}{\sum_{i} k_{i}}$, where the $k_{i}$ s are the rate constants of decay from the chlorophyll excited state (see Appendix B). Chlorophyll fluorescence lifetimes of thylakoid membranes are more complicated because of the large number of chlorophylls that can transfer energy to each other. The interpretation of these lifetimes requires a model of energy transfer in the thylakoid membrane.

Gilmore et al. (1995) fit data from thylakoids with and without $\mathrm{qE}$ to lifetime distributions centered at $400 \mathrm{ps}$ and 2 ns. The amplitude of the 400 ps component was larger in the "qE on" state than in the "qE off" state. Because the lifetimes were conserved between the thylakoids in the two states, the lifetimes were interpreted as "puddles" of PSIIs that cannot transfer energy to one another. Within a puddle, energy transfer was assumed to occur much faster than any of the decay processes. The faster 400 ps component was attributed to PSIIs that had access to a $\mathrm{qE}$ site and was the first assignment of an excited state lifetime for $\mathrm{qE}$. However, the measurement and model were not able to extract detailed information about the energy transfer network with $\mathrm{qE}$ on, such as the location(s) of quenching sites.

Understanding the energy transfer network with $\mathrm{qE}$ on requires a mathematical framework that incorporates that information. The equation describing the changes in excitation population on any node in the network is given by the master equation:

$\frac{\mathrm{d} P(t)}{\mathrm{d} t}=K P(t)$,

where $P(t)$ is a vector containing the populations of each node at a time $t$ and $K$ is a rate matrix that contains all of

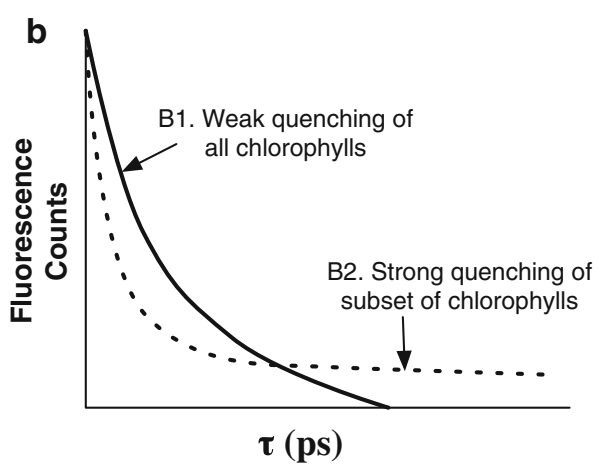

fluorescence yields even thought they display different fluorescence lifetimes. b Illustration of fluorescence lifetimes of moderate quenching of all fluorophores (case B1, solid line) and strong quenching of a small fraction of fluorophores (case B2, dashed line) which cannot be differentiated using fluorescence yield measurements

the information regarding energy transfer connectivity and rates, $\mathrm{qE}$ and $\mathrm{RC}$ quenching rates, and fluorescence and ISC rates. The fluorescence decay $F(t)$ in this formalism is simply the sum of $P(t)$ over all nodes in the network, weighted by the rate of fluorescence at each node (Yang et al. 2003). Knowing $K$ is equivalent to knowing the energy transfer network, and a full understanding of $\mathrm{qE}$ requires characterizing the changes in $K$ between dark- and light-adapted grana membranes (see Fig. 6).

To determine $K$ in grana membranes with qE on, Holzwarth and coworkers measured and fit fluorescence lifetimes on quenched and unquenched leaves with closed RCs of wild type and $n p q 4, n p q 1$, and L17 leaves from A. thaliana. A kinetic model for energy quenching in thylakoid membranes was fit to the fluorescence lifetime data using target analysis (Holzwarth et al. 2009). The kinetic model $(K)$ contained the assumption that all the pigments in the grana membrane are connected, with excitation energy transfer between them occurring much faster than charge separation. The model was first fit to dark-acclimated leaves. Fitting the model with the data from light-acclimated leaves required increasing the nonradiative decay rate of the antenna compartment and including an additional compartment with a decay time of $\sim 400 \mathrm{ps}$. The increase in the non-radiative decay rate correlated positively with the amount of zeaxanthin, and the amplitude of the detached compartment correlated positively with the amount of PsbS. These correlations led to the proposal that there are two mechanisms of $\mathrm{qE}$ : one that was zeaxanthin dependent that occurred in the antenna of the PSII supercomplex, and one that was PsbS dependent that occurred by detachment of LHCII trimers from PSII. A more complex model for energy transfer in the thylakoid membrane compared to that in Gilmore et al. (1995) resulted in more detailed information about the energy transfer network.

It is still unclear what the appropriate model is for describing energy transfer in grana membranes. Recent 
work by van Oort et al. (2010) has suggested that the migration time of excitations in thylakoid membranes makes up $\sim 50 \%$ of the average chlorophyll fluorescence lifetime. This result suggests that models that assume that energy transfer is instantaneous may not be sufficiently detailed to accurately describe energy transfer in grana membranes. Target analysis has been used to determine energy transfer models on a wide range of samples (Holzwarth 1996; van Stokkum et al. 2004). However, the fluorescence lifetime is a coarse-grained measurement, as it is a measure of the sum of all the excitation populations as a function of time. It has recently been shown that different kinetic models can fit fluorescence lifetime data equally well (Tian et al. 2013; van der Weij-de Wit et al. 2011). This means that researchers cannot necessarily differentiate between purely phenomenological models. EM and AFM measurements would allow for the determination of the relative location and orientation of proteins within the thylakoid membrane. Furthermore, the crystal structures of some individual proteins are known, which, when used with EM and AFM images, could allow for a detailed picture of the relative location of chlorophylls in the membrane. An energy transfer model that incorporates both structural information and fluorescence lifetime data would be extremely useful in identifying sites of quenching and the rates with which they quench excitation energy.

\section{Transient Absorption spectroscopy}

Transient absorption (TA) spectroscopy is a method of probing the ultrafast dynamics intermediates involved in the photophysical mechanism of quenching. Unlike fluorescence measurements, TA can detect non-emissive species. TA measures the absorption spectrum of a sample at a fixed time after excitation (Berera et al. 2009). In TA measurements, two pulsed beams, a pump and a probe, are applied to the sample with a fixed time delay between them. The pump beam excites a portion of the chromophores in the sample. The probe beam, which is much weaker, is subsequently transmitted through the sample to measure an absorption spectrum. A difference absorption spectrum $(\Delta A)$ is calculated by subtracting the absorption spectrum of the sample without the pump pulse from the absorption spectrum when the pump pulse has excited the sample. $\Delta A$ can then be measured as a function of wavelength $\lambda$ and the time delay $\tau$ between the pump and probe pulses. The lower limit of $\tau$ is determined by the pulse width of the laser (for ultrafast systems this is on the order of $100 \mathrm{fs}$ ) and the upper limit is determined by the scanning range of the delay stage that controls the delay between the pump and probe pulses (usually around $1 \mathrm{~ns}$ ). $\Delta A(\lambda, \tau)$ is a complex quantity that may have contributions from ground state bleaching (meaning loss of absorption from the ground state), excited state absorption, stimulated emission from the excited state, and absorption from the transfer of excitation to a different molecule than the one that was initially excited. TA spectroscopy has been used to observe absorption from non-emissive intermediate states involved in $\mathrm{qE}$ after excitation of chlorophyll in photosynthetic proteins and thylakoid membranes.

Many groups have investigated the photophysical mechanism of qE quenching using TA spectroscopy. A large number of TA studies have been carried out examining isolated pigment-protein complexes (e.g., El-Samad et al. 2006; Müller et al. 2010; Ruban et al. 2007) as well as synthetic constructs that mimic $\mathrm{qE}$ in artificial systems (e.g., Berera et al. 2006; Terazono et al. 2011); a full discussion of these studies is outside the scope of this paper. Because the site of $\mathrm{qE}$ may not be localized on a single protein, and because the quenching properties of proteins may be altered when they are isolated from the membrane environment, correlating the results of TA experiments with $\mathrm{qE}$ quenching in isolated proteins is difficult. As a result, it has been necessary to study intact systems that are capable of performing qE. Thylakoid membranes are the smallest isolatable units that are capable of activating $\mathrm{qE}$ with light and provide a system that can be studied in solution, unlike solid-state samples such as leaves. Experiments on thylakoid membranes (Holt et al. 2005; Ma et al. 2003) have suggested that carotenoids are directly involved in the $\mathrm{qE}$ mechanism. Recently, a method for measuring TA during light adaptation in intact leaves was developed by the Holzwarth group (2013), which holds great promise for examining the photophysical mechanism of $\mathrm{qE}$ in intact photosynthetic systems.

The results of TA spectroscopy, sometimes accompanied by theoretical calculations, have led to the proposal of several different hypotheses for the photophysical mechanism of the deactivation of excited singlet chlorophyll via qE quenching: (1) the aggregation of LHCII leading to quenching by energy transfer to the lutein $S_{1}$ state (Pascal et al. 2005; Ruban et al. 2007); (2) excitonic coupling between zeaxanthin and chlorophyll, leading to dissipation of energy via the zeaxanthin $S_{1}$ state (Bode et al. 2009), which has also been recently observed in reconstituted proteoliposomes containing PsbS and LHCII (Wilk et al. 2013); (3) aggregation of the LHCII trimers leading to chlorophyll-chlorophyll charge-transfer state that facilities quenching (Müller et al. 2010), which has also been correlated with a red-shifted emission of chlorophyll fluorescence (Holzwarth et al. 2009); and (4) the formation of a chlorophyll-zeaxanthin charge-transfer state that quenches chlorophyll fluorescence (Ahn et al. 2008; Holt et al. 2005). These hypotheses are not mutually exclusive, but confirming or eliminating any one of them is challenging. These challenges arise from the large number of 
chromophores in the membrane and the lack of spectral separation between different species. For instance, chlorophyll radical cations and anions do not have distinct, sharp spectral peaks (Fujita et al. 1978), making it difficult to unambiguously prove or disprove the formation of chlorophyll radical species during qE. Carotenoid cations do have distinct spectral peaks in the wavelength range of 900-1,000 nm (Galinato et al. 2007) and have been observed to be correlated with $\mathrm{qE}$ in isolated thylakoid membranes (Holt et al. 2005) and isolated complexes (Ahn et al. 2008; Avenson et al. 2008). Moving forward, it seems likely that correlating the amplitudes and dynamics of TA experiments with $\mathrm{qE}$ in vivo will be necessary for differentiating between different $\mathrm{qE}$ mechanisms.

\section{New tools for characterizing $\mathrm{qE}$ in vivo}

Since the first discovery of $\mathrm{qE}$ quenching, a great deal of information has been revealed about the triggers, components, and spectroscopic signatures associated with $\mathrm{qE}$. Measurements of chloroplasts, isolated thylakoids, and isolated proteins have yielded numerous hypotheses regarding the trigger, site, and photophysical mechanisms of $\mathrm{qE}$. In our view, resolving the many hypotheses that have been proposed based on isolated systems requires the development of techniques to study $\mathrm{qE}$ in intact living systems such as whole leaves and live algae.
Because $\mathrm{qE}$ is a dynamic process, a full understanding requires knowledge of the timescales of constituent processes. Interpretation of results in intact systems is complicated because the events leading up to $\mathrm{qE}$ occur on many timescales and are affected by a large number of dynamic processes. Figure 8 illustrates the range of timescales involved in $\mathrm{qE}$. In particular, the timescale of the appearance of $\mathrm{qE}$ quenching, as observed by fluorescence measurements, is a combination of the formation of the triggers (the lumen $\mathrm{pH}$ and $\Delta \mathrm{pH}$ ) and the timescale and set points of the membrane rearrangements (e.g., protein activations, protein aggregation) that give rise to the formation of $\mathrm{qE}$. The lumen $\mathrm{pH}$ is itself determined by four processes: (1) water splitting at PSII, (2) proton pumping at cytochrome $b_{6} f$, (3) proton efflux through ATP synthesis, and (4) parsing of the proton motive force into a $\Delta \mathrm{pH}$ and a $\Delta \psi$ component by the motion of ions across the thylakoid membrane.

The multitude of interconnected processes that give rise to a $\mathrm{qE}$ quenching state makes it difficult to differentiate between mechanistic hypotheses. To address this difficulty, we have developed a kinetic model of the processes in photosynthesis that give rise to $\mathrm{qE}$. Our model, which is inspired by state-space models of engineering control theory analysis (Eberhard et al. 2008), calculates the lumen $\mathrm{pH}$ and simulates the induction and relaxation of $\mathrm{qE}$ in low and high light intensity (Zaks et al. 2012). The model currently consists of 24 non-linear differential equations
Fig. 8 Schematic of feedback loop governing $\mathrm{qE}$ (solid black rectangles), and the broad range of timescales of processes giving rise to $\mathrm{qE}$ (dashed colored rectangles)

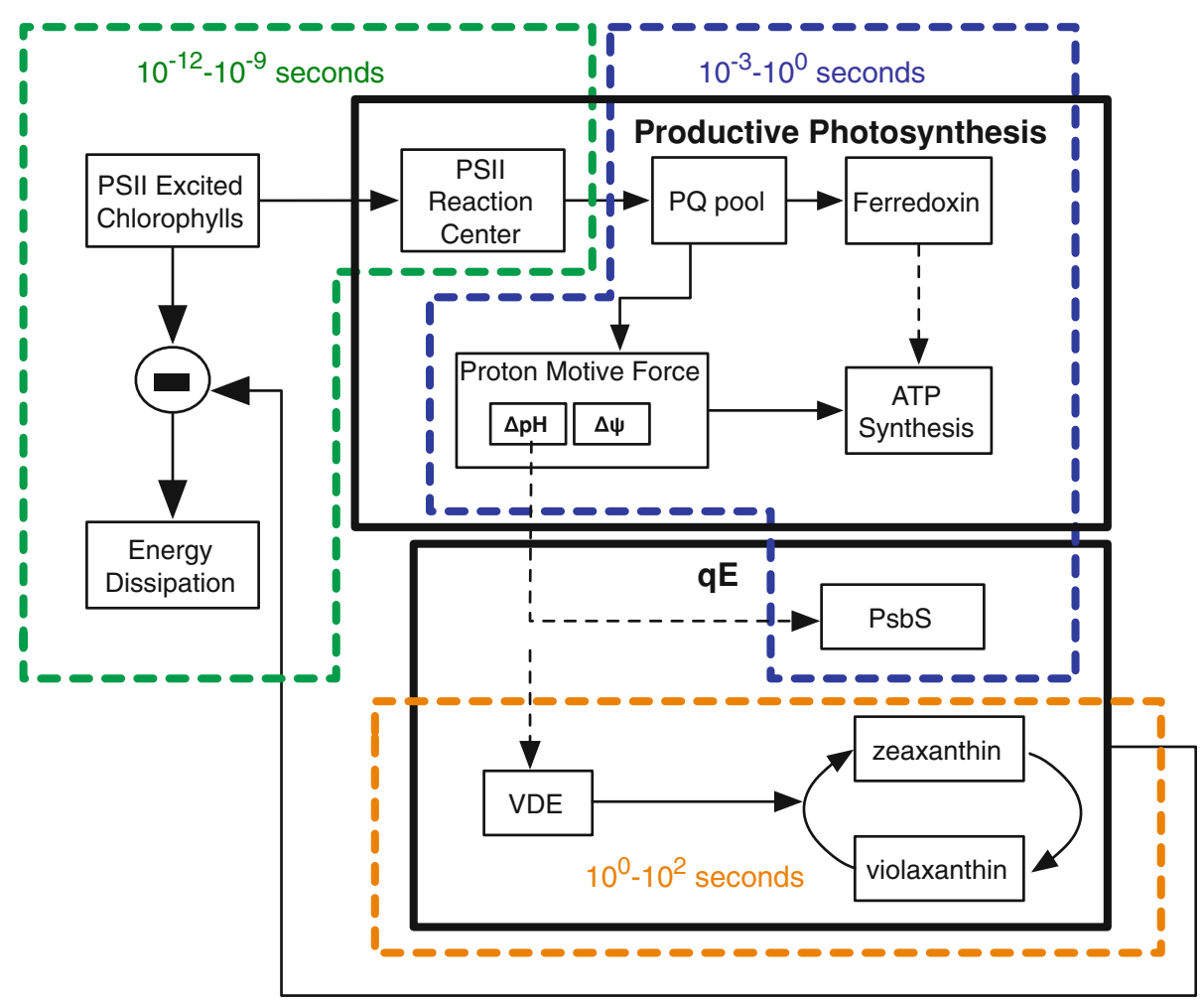


Fig. 9 Comparison between systems model and measured $\mathrm{qE}$ component of NPQ in a low light intensity and $\mathbf{b}$ high light intensity. (adapted from Zaks et al. 2012)
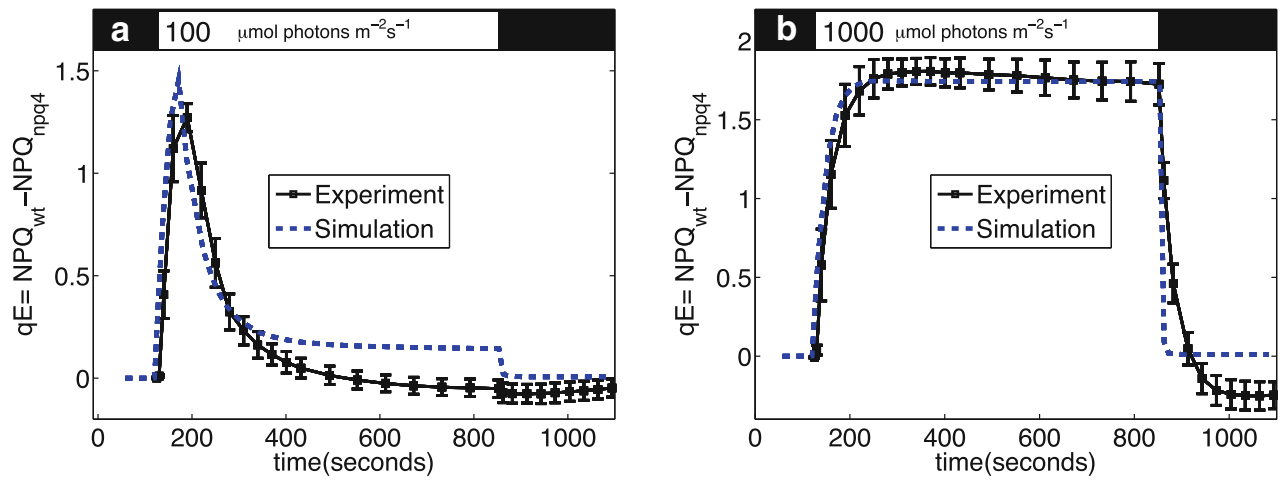

that calculate the $\mathrm{pH}$ in the lumen on timescales ranging from microseconds to minutes.

We tested the effectiveness of the model by calculating chlorophyll fluorescence yields and comparing those predictions to PAM fluorescence measurements. We quantified the $\mathrm{qE}$ component of NPQ derived from PAM traces by subtracting the amount of NPQ developed in wild type mutant from the amount of NPQ developed in the $n p q 4$ mutant. Figure 9 shows a comparison between the predictions of the model and experimental measurements of rapidly reversible NPQ. The model shows good agreement with measurements of $\mathrm{qE}$ at 100 and $1,000 \mu \mathrm{mol}$ photons $\mathrm{m}^{-2} \mathrm{~s}^{-1}$ (Zaks et al. 2012)

A benefit of using kinetic models in studying $\mathrm{qE}$ mechanism is that they make it possible to separate different processes giving rise to $\mathrm{qE}$. For example, the timescale of $\mathrm{qE}$ appearance, as observed by PAM or fluorescence lifetime measurements, is affected by both the timescale of the formation of the $\Delta \mathrm{pH}$ and by the dynamics of the membrane rearrangement following $\mathrm{qE}$ triggering. A mathematical model such as the one we developed (Zaks et al. 2012) provides a framework for testing hypotheses of many mechanisms relating to $\mathrm{qE}$. For instance, it is not clear whether the $\mathrm{pH}$-sensing components of the membrane have a fixed $\mathrm{p} K_{\mathrm{a}}$, as assumed in Takizawa et al. (2007), or have a variable $\mathrm{p} K_{\mathrm{a}}$, as proposed in Johnson and Ruban (2011) and Johnson et al. (2012). It is possible to quantify these two hypotheses using mathematical expressions, then integrate both expressions into the model and compare the predictions of either hypothesis. Additionally, as mathematical models of individual components are developed and refined, these models could be integrated in a modular fashion into the framework of a systems model to test the implications of a detailed understanding on the behavior of the thylakoid system as a whole. To aid this effort, we have made the documented MATLAB code of our model available (Zaks). We have also created a GUI for our model that facilitates the exploration of the model by researchers from a broad range of backgrounds (Zaks 2012).
A challenge associated with experimentally testing the predictions of kinetic models is that methods for measuring $\mathrm{qE}$ typically measure either slow biochemical changes (sec to min timescale, which can be characterized using PAM) or the fast dynamics in the light-harvesting antenna (fs to ns timescale, by measuring fluorescence lifetimes or TA) in dark- or light-acclimated samples. Understanding how the triggers/components of $\mathrm{qE}$ act in concert to activate quenching requires a technique that bridges both slow and fast timescales. The photophysical mechanisms and sites involved in $\mathrm{qE}$ are intimately tied to the biochemical and physical changes that occur to activate these mechanisms.

To fill this gap in techniques for measuring $\mathrm{qE}$, we have developed a technique for measuring the changing fluorescence lifetime as $\mathrm{qE}$ turns on in plants and algae, which we call "fluorescence lifetime snapshots" (Fig. 10) (Amarnath et al. 2012). It is a two-dimensional (2D) technique with one time axis being the fluorescence decay time and the second being the adaptation/relaxation timescale. The technique has so far been used to measure the changes in fluorescence lifetimes in live cells of Chlamydomonas reinhardtii during the transition from a dark- to a lightacclimated state, and back to a dark-acclimated state. The data from the measurement on algae were globally fit to three exponential decays. This result suggested that the three lifetimes could be treated as separate pools of PSII that cannot transfer between each other. Two of the populations had lifetimes of 65 and 305 ps, with the third having a lifetime of $1 \mathrm{~ns}$. The amplitudes of the two shorter lifetimes increased during the light treatment and decreased in the ensuing darkness. In addition, these amplitudes substantially decreased when the $\mathrm{pH}$ gradient was dissipated using nigericin. The amplitudes associated with the 65 and 305 ps lifetime components exhibited different dynamics during $\mathrm{qE}$ induction and relaxation, which led us to suggest that there are two different mechanisms associated with $\mathrm{qE}$ in $C$. reinhardtii. This technique correlates the $T$ axis, which describes the timescales of qE triggering, with the $t$ axis, which probes changes in the membrane and photophysical mechanism of $\mathrm{qE}$. 


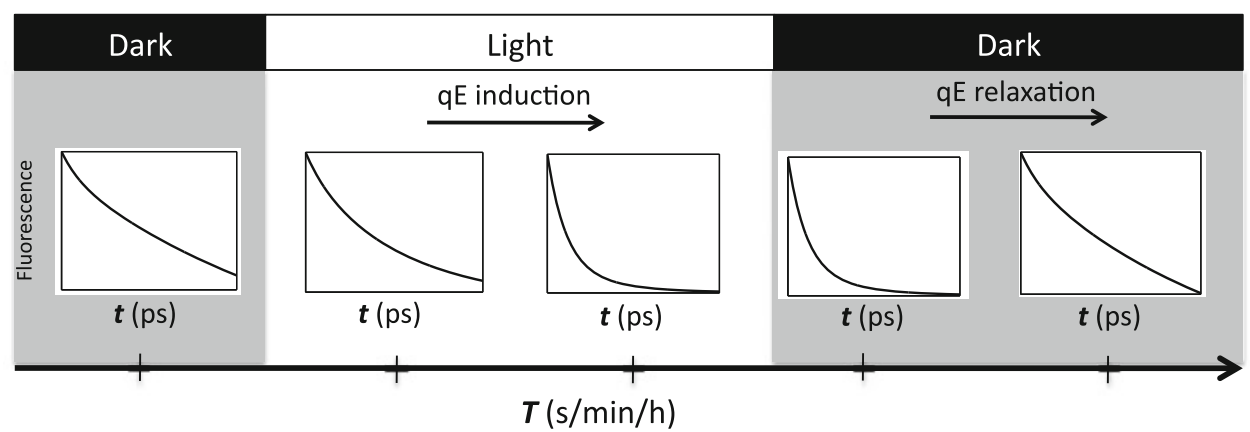

Fig. 10 Schematic of "fluorescence lifetime snapshots" measurements. The technique tracks changes on both the $T$ timescale (sec to hours) as well as in the $t$ timescale (ps to ns). qE triggering and the thylakoid membrane rearrangement occur on the $T$ timescale. Quenching of chlorophyll fluorescence occurs on the $t$ timescale and contains information about the membrane configuration
As discussed in the "Fluorescence lifetimes" section and Appendix B, the insight from fitting fluorescence lifetimes to multiple exponential decays is limited. Using the fluorescence lifetime snapshot measurements to differentiate between different hypotheses for $\mathrm{qE}$ mechanisms requires fitting the fluorescence lifetimes to a detailed mechanistic model of energy transfer. Because different energy transfer models are able to fit fluorescence lifetime data well (van der Weij-de Wit et al. 2011), much theoretical and experimental progress remains to be made in developing accurate models of energy transfer in PSII. We are optimistic that future developments in this area will enable the interpretation of fluorescence lifetime snapshots in the context of a mechanistic model for $\mathrm{qE}$.

\section{Concluding remarks}

Looking forward, much progress in the development of experimental techniques and theoretical models will be needed before the site(s) and mechanism(s) of $\mathrm{qE}$ are identified and the triggering processes and ensuing membrane changes are characterized. Obtaining unambiguous answers is particularly challenging because the pigments and proteins involved in $\mathrm{qE}$ are found inside of a lipid membrane, are buried within a cell, are highly dependent on interactions with their local environment, and undergo changes on a wide range of timescales. To address these challenges, it will be necessary to develop experimental techniques with high spatial, spectral, and temporal resolution that can probe dense, heterogeneous membranebased systems. It is also becoming possible, and will likely be necessary, to develop mathematical models that take advantage of increasingly powerful computing power to encompass the true complexity of qE. It will be important that these models be capable of making falsifiable predictions that enable differentiation between different mechanisms of qE. Such developments should provide valuable, as understanding a detailed mechanism of $\mathrm{qE}$ would profoundly extend our understanding of the regulation of biological energy transduction and will likely provide useful design principles for the regulation of light harvesting in fluctuating light conditions.

Acknowledgments We thank Matt Brooks, Alizée Malnoë, and Anna Schneider for helpful comments on the manuscript and Doran Bennett and Eleonora De Re for helpful discussions. This work was supported by the Director, Office of Science, Office of Basic Energy Sciences of the US Department of Energy under Contract DEAC0205CH11231 and the Division of Chemical Sciences, Geosciences, and Biosciences, Office of Basic Energy Sciences of the US Department of Energy through Grant DE-AC03-76SF000098. EJ S-G was supported by a National Science Foundation Graduate Research Fellowship.

Open Access This article is distributed under the terms of the Creative Commons Attribution License which permits any use, distribution, and reproduction in any medium, provided the original author(s) and the source are credited.

\section{Appendix A: Pulse amplitude modulated fluorescence}

A typical PAM trace of a wild type leaf of A. thaliana is shown in Fig. 2. At the beginning of the PAM trace, the actinic light source is off. Then, a 1-s saturating flash is applied, and the maximum fluorescence measured during the flash is called $F_{\mathrm{m}}$. Using a simplified definition of chlorophyll quantum yield described in Ahn et al. (2009) and Hendrickson et al. (2005), we can write $F_{\mathrm{m}}$ as

$F_{\mathrm{m}} \propto \Phi_{F, F_{\mathrm{m}}}=\frac{k_{\mathrm{F}}}{k_{\mathrm{F}}+k_{\mathrm{IC}}+k_{\mathrm{ISC}}}$,

where $\Phi_{F, F_{\mathrm{m}}}$ is the fluorescence quantum yield during the measurement of $F_{\mathrm{m}}$ and $k_{\mathrm{F}}, k_{\mathrm{IC}}$, and $k_{\mathrm{ISC}}$ are the rate constants of decay for fluorescence, internal conversion, and intersystem crossing, respectively (Ahn et al. 2009). There, rate constant for photochemistry at the RC in the 
denominator is equal to 0 because the saturating pulse closes all RCs and temporarily blocks photochemistry. After the actinic light, bar at top of plot, is turned on, a saturating pulse is applied every minute. The actinic light remains on for $10 \mathrm{~min}$, followed by darkness for $10 \mathrm{~min}$. The maximum fluorescence yield during each of these pulses is called $F_{\mathrm{m}}^{\prime}$,

$F_{\mathrm{m}}^{\prime} \propto \Phi_{F, F_{\mathrm{m}}^{\prime}}=\frac{k_{\mathrm{F}}}{k_{\mathrm{NPQ}}(T)+k_{\mathrm{F}}+k_{\mathrm{IC}}+k_{\mathrm{ISC}}}$,

where $k_{\mathrm{NPQ}}$ is the rate constant for dissipation by NPQ. Note that $F_{\mathrm{m}}^{\prime}$ is time dependent because $k_{\mathrm{NPQ}}$ is time dependent.

There are many ways to extract the dynamics and the amplitude of the $\mathrm{qE}$ component of quenching from a PAM trace. One way is by measuring the fluorescence after $\mathrm{qE}$ has relaxed (with other components of NPQ such as qI and qT still intact); called $F_{\mathrm{m}}^{\prime \prime}$, it is possible to estimate the amount of $\mathrm{qE}$ (Demmig and Winter 1988):

$\mathrm{qE}=\frac{F_{\mathrm{m}}^{\prime \prime}-F_{\mathrm{m}}^{\prime}}{F_{\mathrm{m}}^{\prime \prime}}$.

This qE parameter can be used to see what components or chemicals affect the amplitude of $\mathrm{qE}$ (Johnson and Ruban 2011). Additionally, it is possible to estimate the quantum yield of $\mathrm{qE}, \Phi_{\mathrm{qE}}$. by additionally measuring $F_{\mathrm{S}}$, the fluorescence yield, immediately before a saturating pulse is applied.

$\Phi_{\mathrm{qE}}=\frac{F_{\mathrm{m}}^{\prime \prime}-F_{\mathrm{m}}^{\prime}}{F_{\mathrm{m}}^{\prime \prime}} \frac{F_{\mathrm{S}}}{F_{\mathrm{m}}^{\prime}}$

where $F_{\mathrm{S}}$ is the fluorescence of the PAM trace right before a saturating pulse is applied (Ahn et al. 2009).

\section{Appendix B: Time-correlated single photon counting}

In this section, we describe the basic principles of TCSPC. A short pulse of light is used to excite a fluorophore such as chlorophyll. Free chlorophyll in solution in the excited state can relax back to the ground state via fluorescence, IC, or ISC. The rate constant for each decay process does not depend on the time that the chlorophyll has been in the excited state. A photon of fluorescence is detected at time $t+\Delta t$ after excitation. The experiment is repeated many times, with many photons of fluorescence observed and binned (with bin width equal to $\Delta t$ ) to make a histogram. This histogram has a shape defined by the probability $P(t)$ that the chlorophyll molecule is in the excited state at time $t=M \Delta t$. If, after a $\Delta t$ timestep, the probability that the chlorophyll molecule is still in the excited state is $1-\left(k_{\mathrm{F}}+k_{\mathrm{IC}}+k_{\mathrm{ISC}}\right) \Delta t$, it follows that
$P(t)=\left(1-\left(k_{\mathrm{F}}+k_{\mathrm{IC}}+k_{\mathrm{ISC}}\right) \frac{t}{M}\right)^{M}$

In the limit that $\Delta t$ goes to 0 , or $M$ goes to infinity,

$$
\begin{aligned}
P(t) & =\lim _{M \rightarrow \infty}\left(1-\left(k_{\mathrm{F}}+k_{\mathrm{IC}}+k_{\mathrm{ISC}}\right) \frac{t}{M}\right)^{M} \\
& =\exp \left(\frac{-t}{k_{\mathrm{F}}+k_{\mathrm{IC}}+k_{\mathrm{ISC}}}\right) .
\end{aligned}
$$

The form of the decay of the population of chlorophyll excited states goes as an exponential with a time constant $\tau=\frac{1}{k_{\mathrm{F}}+k_{\mathrm{IC}}+k_{\mathrm{ISC}}}$.

The width of the light pulse and the response time of the instrument are convolved with the fluorescence decay of the sample. To extract the decay, $F(t)$ (analogous to $P(t)$ above), requires a reconvolution fit of the data $I(t)$,

$I(t)=\int_{-\infty}^{t} \operatorname{IRF}\left(t^{\prime}\right) \sum_{i}^{n} A_{i} \mathrm{e}^{\frac{-t-t^{\prime}}{\tau_{i}}}$,

where IRF is the instrument response function. It is often assumed that $F(t)=\sum_{i}^{n} A_{i} \mathrm{e}^{\frac{-t}{\tau_{i}}}$, where $n$ is the number of exponentials, $A_{i}$ are the amplitudes, and $\tau_{i}$ are the lifetimes. $A_{i}$ and $\tau_{i}$ are fit to the data using a criterion such as leastsquares or maximum likelihood (Lakowicz 2006). Measurements of the fluorescence lifetime of the chlorophyll in the thylakoid membrane exhibit more complicated decay dynamics (see Fluorescence lifetimes section).

\section{References}

Ahn TK, Avenson TJ, Ballottari M, Cheng YC, Niyogi KK, Bassi R, Fleming GR (2008) Architecture of a charge-transfer state regulating light harvesting in a plant antenna protein. Science 320(5877):794-797

Ahn TK, Avenson TJ, Peers G, Li Z, Dall'Osto L, Bassi R, Niyogi KK, Fleming GR (2009) Investigating energy partitioning during photosynthesis using an expanded quantum yield convention. Chem Phys 357(1-3):151-158

Amarnath K, Zaks J, Park SD, Niyogi KK, Fleming GR (2012) Fluorescence lifetime snapshots reveal two rapidly reversible mechanisms of photoprotection in live cells of Chlamydomonas reinhardtii. Proc Natl Acad Sci USA 109(22):8405-8410

Andersson J, Walters RG, Horton P, Jansson S (2001) Antisense inhibition of the photosynthetic antenna proteins CP29 and CP26: implications for the mechanism of protective energy dissipation. Plant Cell 13(5):1193-1204

Avenson TJ, Ahn TK, Zigmantas D, Niyogi KK, Li Z, Ballottari M, Bassi R, Fleming GR (2008) Zeaxanthin radical cation formation in minor light-harvesting complexes of higher plant antenna. J Biol Chem 283(6):3550-3558

Bailleul B, Cardol P, Breyton C, Finazzi G (2010) Electrochromism: a useful probe to study algal photosynthesis. Photosynth Res 106(1-2):179-189

Baker NR (2008) Chlorophyll fluorescence: a probe of photosynthesis in vivo. Annu Rev Plant Biol 59:89-113 
Barber J (1994) Molecular basis of the vulnerability of photosystem II to damage by light. Aust J Plant Physiol 22:201-208

Beddard G, Porter G (1976) Concentration quenching in chlorophyll. Nature 260(5549):366-367

Berera R, Herrero C, Van Stokkum IHM, Vengris M, Kodis G, Palacios RE, Van Amerongen H, Van Grondelle R, Gust D, Moore TA, Moore AL, Kennis JTM (2006) A simple artificial light-harvesting dyad as a model for excess energy dissipation in oxygenic photosynthesis. Proc Natl Acad Sci USA 103(14): 5343-5348

Berera R, van Grondelle R, Kennis JTM (2009) Ultrafast transient absorption spectroscopy: principles and application to photosynthetic systems. Photosynth Res 101(2-3):105-118

Betterle N, Ballottari M, Zorzan S, de Bianchi S, Cazzaniga S, Dall'Osto L, Morosinotto T, Bassi R (2009) Light-induced dissociation of an antenna hetero-oligomer is needed for non-photochemical quenching induction. J Biol Chem 284(22):15255-15266

Blankenship RE (2002) Molecular mechanisms of photosynthesis. Blackwell Science, Oxford

Bode S, Quentmeier CC, Liao PN, Hafi N, Barros T, Wilk L, Bittner F, Walla PJ (2009) On the regulation of photosynthesis by excitonic interactions between carotenoids and chlorophylls. Proc Natl Acad Sci USA 106(30):12311-12316

Boekema EJ, van Breemen JF, van Roon H, Dekker JP (2000) Arrangement of photosystem II supercomplexes in crystalline macrodomains within the thylakoid membrane of green plant chloroplasts. J Mol Biol 301(5):1123-1133

Briantais JM, Vernotte C, Picaud M, Krause GH (1979) A quantitative study of the slow decline of chlorophyll a fluorescence in isolated chloroplasts. Biochim Biophys Acta 548(1):128-138

Brooks MD, Niyogi KK (2011) Use of a pulse-amplitude modulated chlorophyll fluorometer to study the efficiency of photosynthesis in Arabidopsis plants. Methods Mol Biol 775:299-310

Caffarri S, Kouřil R, Kereiche S, Boekema EJ, Croce R (2009) Functional architecture of higher plant photosystem II supercomplexes. EMBO J 28(19):3052-3063

Clayton RK, Szuts EZ, Fleming H (1972) Photochemical electron transport in photosynthetic reaction centers from Rhodopseudomonas spheroides. 3. Effects of orthophenanthroline and other chemicals. Biophys J 12(1):64-79

Crimi M, Dorra D, Bösinger CS, Giuffra E, Holzwarth AR, Bassi R (2001) Time-resolved fluorescence analysis of the recombinant photosystem II antenna complex CP29. Eur J Biochem 268(2): 260-267

Croce R, van Amerongen H (2011) Light-harvesting and structural organization of photosystem II: from individual complexes to thylakoid membrane. J Photochem Photobiol B 104(1-2):142-153

Cruz J, Sacksteder C, Kanazawa A, Kramer D (2001) Contribution of electric field $(\Delta \psi)$ to steady-state transthylakoid proton motive force (pmf) in vitro and in vivo. Control of pmf parsing into $\Delta \psi$ and $\Delta \mathrm{pH}$ by ionic strength. Biochemistry 40(5):1226-1237

Dall'Osto L, Lico C, Alric J, Giuliano G, Havaux M, Bassi R (2006) Lutein is needed for efficient chlorophyll triplet quenching in the major LHCII antenna complex of higher plants and effective photoprotection in vivo under strong light. BMC Plant Biol 6(1):32

Daum B, Nicastro D, Austin J, McIntosh JR, Kühlbrandt W (2010) Arrangement of photosystem II and ATP synthase in chloroplast membranes of spinach and pea. Plant Cell 22(4):1299-1312

de Bianchi S, Dall'Osto L, Tognon G, Morosinotto T, Bassi R (2008) Minor antenna proteins CP24 and CP26 affect the interactions between photosystem II subunits and the electron transport rate in grana membranes of Arabidopsis. Plant Cell 20(4):1012-1028

de Bianchi S, Betterle N, Kouril R, Cazzaniga S, Boekema E, Bassi R, Dall'Osto L (2011) Arabidopsis mutants deleted in the lightharvesting protein Lhcb4 have a disrupted photosystem II macrostructure and are defective in photoprotection. Plant Cell 23(7):2659-2679

De Carlo S, El-Bez C, Alvarez-Rúa C, Borge J, Dubochet J (2002) Cryo-negative staining reduces electron-beam sensitivity of vitrified biological particles. J Struct Biol 138(3):216-226

Dekker JP, Boekema EJ (2005) Supramolecular organization of thylakoid membrane proteins in green plants. Biochim Biophys Acta 1706(1-2):12-39

Demmig-Adams B (1990) Carotenoids and photoprotection in plants: a role for the xanthophyll zeaxanthin. Biochim Biophys Acta 1020(1):1-24

Demmig-Adams B, Winter K (1988) Characterisation of three components of non-photochemical fluorescence quenching and their response to photoinhibition. Aust J Plant Physiol 15(2):163

Dominici P, Caffarri S, Armenante F, Ceoldo S, Crimi M, Bassi R (2002) Biochemical properties of the PsbS subunit of photosystem II either purified from chloroplast or recombinant. J Biol Chem 277(25):22750-22758

Drepper F, Carlberg I, Andersson B, Haehnel W (1993) Lateral diffusion of an integral membrane protein: Monte Carlo analysis of the migration of phosphorylated light-harvesting complex II in the thylakoid membrane. Biochemistry 32(44):11915-11922

Duffy CDP, Johnson MP, Macernis M, Valkunas L, Barford W, Ruban AV (2010) A theoretical investigation of the photophysical consequences of major plant light-harvesting complex aggregation within the photosynthetic membrane. J Phys Chem B 114(46):15244-15253

Durrant J, Giorgi L, Barber J, Klug D, Porter G (1990) Characterisation of triplet states in isolated photosystem II reaction centres: oxygen quenching as a mechanism for photodamage. Biochim Biophys Acta 1017(2):167-175

Eberhard S, Finazzi G, Wollman FA (2008) The dynamics of photosynthesis. Annu Rev Genet 42:463-515

El-Samad H, Prajna S, Papachristodoulou A, Doyle J, Khammash M (2006) Advanced methods and algorithms for biological networks analysis. Proc IEEE 94(4):832-853

Fuciman M, Enriquez MM, Polívka T, Dall'Osto L, Bassi R, Frank HA (2012) Role of xanthophylls in light harvesting in green plants: a spectroscopic investigation of mutant LHCII and Lhcb pigment-protein complexes. J Phys Chem B 116(12):3834-3849

Fujita I, Davis M, Fajer J (1978) Anion radicals of pheophytin and chlorophyll a: their role in the primary charge separations of plant photosynthesis. J Am Chem Soc 100(19):6280-6282

Fukuma T, Higgins MJ, Jarvis SP (2007) Direct imaging of individual intrinsic hydration layers on lipid bilayers at angstrom resolution. Biophys J 92(10):3603-3609

Funk C, Schröder WP, Napiwotzki A, Tjus SE, Renger G, Andersson B (1995) The PSII-S protein of higher plants: a new type of pigment-binding protein. Biochemistry 34(35):11133-11141

Galinato MGI, Niedzwiedzki D, Deal C, Birge RR, Frank HA (2007) Cation radicals of xanthophylls. Photosynth Res 94(1):67-78

Gilmore A, Hazlett T, Govindjee (1995) Xanthophyll cycle-dependent quenching of photosystem II chlorophyll a fluorescence: formation of a quenching complex with a short fluorescence lifetime. Proc Natl Acad Sci USA 92(6):2273-2277

Goss R, Opitz C, Lepetit B, Wilhelm C (2008) The synthesis of NPQeffective zeaxanthin depends on the presence of a transmembrane proton gradient and a slightly basic stromal side of the thylakoid membrane. Planta 228(6):999-1009

Govindjee (2004) Chlorophyll $a$ fluorescence: a bit of basics and history. In: Papageorgiu GC, Govindjee (eds) Chlorophyll a fluorescence: a signature of photosynthesis, advances in photosynthesis and respiration, vol 19. Springer, Dordrecht

Havaux M, Dall'osto L, Bassi R (2007) Zeaxanthin has enhanced antioxidant capacity with respect to all other xanthophylls in 
Arabidopsis leaves and functions independent of binding to PSII antennae. Plant Physiol 145(4):1506-1520

Heldt WH, Werdan K, Milovancev M, Geller G (1973) Alkalization of the chloroplast stroma caused by light-dependent proton flux into the thylakoid space. Biochim Biophys Acta 314(2):224-241

Hendrickson L, Förster B, Pogson BJ, Chow WS (2005) A simple chlorophyll fluorescence parameter that correlates with the rate coefficient of photoinactivation of photosystem II. Photosynth Res 84(1-3):43-49

Holt NE, Zigmantas D, Valkunas L, Li XP, Niyogi KK, Fleming GR (2005) Carotenoid cation formation and the regulation of photosynthetic light harvesting. Science 307(5708):433-436

Holzwarth AR (1996) Data analysis of time-resolved measurements. In: Amesz J, Hoff AJ (eds) Biophysical techniques in photosynthesis, advances in photosynthesis and respiration, vol 26. Kluwer Academic Publishers, Dordrecht

Holzwarth AR, Miloslavina Y, Nilkens M, Jahns P (2009) Identification of two quenching sites active in the regulation of photosynthetic light-harvesting studied by time-resolved fluorescence. Chem Phys Lett 483(4-6):262-267

Holzwarth AR, Lenk D, Jahns P (2013) On the analysis of nonphotochemical chlorophyll fluorescence quenching curves: I. Theoretical considerations. Biochim Biophys Acta 1827(6):786-792

Jahns P, Latowski D, Strzalka K (2009) Mechanism and regulation of the violaxanthin cycle: the role of antenna proteins and membrane lipid. Biochim Biophys Acta 1787(1):3-14

Johnson MP, Ruban AV (2009) Photoprotective energy dissipation in higher plants involves alteration of the excited state energy of the emitting chlorophyll(s) in the light harvesting antenna II (LHCII). J Biol Chem 284(35):23592-23601

Johnson MP, Ruban AV (2010) Arabidopsis plants lacking PsbS protein possess photoprotective energy dissipation. Plant J 61(2):283-289

Johnson MP, Ruban AV (2011) Restoration of rapidly reversible photoprotective energy dissipation in the absence of PsbS protein by enhanced $\Delta \mathrm{pH}$. J Biol Chem 286(22):19973-19981

Johnson MP, Goral TK, Duffy CDP, Brain APR, Mullineaux CW, Ruban AV (2011) Photoprotective energy dissipation involves the reorganization of photosystem II light-harvesting complexes in the grana membranes of spinach chloroplasts. Plant Cell 23(4): 1468-1479

Johnson MP, Zia A, Ruban AV (2012) Elevated $\Delta$ pH restores rapidly reversible photoprotective energy dissipation in Arabidopsis chloroplasts deficient in lutein and xanthophyll cycle activity. Planta 235(1):193-204

Junge WW, Ausländer WW, McGeer AJA, Runge TT (1979) The buffering capacity of the internal phase of thylakoids and the magnitude of the $\mathrm{pH}$ changes inside under flashing light. Biochim Biophys Acta 546(1):121-141

Kirchhoff H, Tremmel I, Haase W, Kubitscheck U (2004) Supramolecular photosystem II organization in grana thylakoid membranes: evidence for a structured arrangement. Biochemistry 43(28):9204-9213

Kirchhoff H, Haferkamp S, Allen JF, Epstein DBA, Mullineaux CW (2008a) Protein diffusion and macromolecular crowding in thylakoid membranes. Plant Physiol 146(4):1571-1578

Kirchhoff H, Lenhert S, Büchel C, Chi L, Nield J (2008b) Probing the organization of photosystem II in photosynthetic membranes by atomic force microscopy. Biochemistry 47(1):431-440

Kiss AZ, Ruban AV, Horton P (2008) The PsbS protein controls the organization of the photosystem II antenna in higher plant thylakoid membranes. J Biol Chem 283(7):3972-3978

Kouřil R, Oostergetel GT, Boekema EJ (2011) Fine structure of granal thylakoid membrane organization using cryo electron tomography. Biochim Biophys Acta 1807(3):368-374

Kouřil R, Wientjes E, Bultema JB, Croce R, Boekema EJ (2012a) High-light vs. low-light: effect of light acclimation on photosystem II composition and organization in Arabidopsis thaliana. Biochim Biophys Acta 1827(3):411-419

Kouřil R, Dekker JP, Boekema EJ (2012b) Supramolecular organization of photosystem II in green plants. Biochim Biophys Acta 1817(1):2-12

Krause GH (1973) The high-energy state of the thylakoid system as indicated by chlorophyll fluorescence and chloroplast shrinkage. Biochim Biophys Acta 292(3):715-728

Krause G, Weis E (1991) Chlorophyll fluorescence and photosynthesis: the basics. Annu Rev Plant Biol 42(1):313-349

Kulheim C, Agren J, Jansson S (2002) Rapid regulation of light harvesting and plant fitness in the field. Science 297(5578): 91-93

Lakowicz JR (2006) Principles of fluorescence spectroscopy, 3rd edn. Springer, New York

Li XP, Bjorkman O, Shih C, Grossman AR, Rosenquist M, Jansson S, Niyogi KK (2000) A pigment-binding protein essential for regulation of photosynthetic light harvesting. Nature 403(6768): 391-395

Li XP, Muller-Moule P, Gilmore AM, Niyogi KK (2002a) PsbSdependent enhancement of feedback de-excitation protects photosystem II from photoinhibition. Proc Natl Acad Sci USA 99(23): 15222-15227

Li XP, Phippard A, Pasari J, Niyogi KK (2002b) Structure-function analysis of photosystem II subunit S (PsbS) in vivo. Funct Plant Biol 29(10):1131-1139

Liu LN, Sturgis JN, Scheuring S (2011) Native architecture of the photosynthetic membrane from Rhodobacter veldkampii. J Struct Biol 173(1):138-145

Ma YZ, Holt NE, Li XP, Niyogi KK, Fleming GR (2003) Evidence for direct carotenoid involvement in the regulation of photosynthetic light harvesting. Proc Natl Acad Sci USA 100(8): 4377-4382

Melis A (1999) Photosystem-II damage and repair cycle in chloroplasts: what modulates the rate of photodamage in vivo. Trends Plant Sci 4(4):130-135

Miloslavina Y, Wehner A, Lambrev PH, Wientjes E, Reus M, Garab G, Croce R, Holzwarth AR (2008) Far-red fluorescence: a direct spectroscopic marker for lhcII oligomer formation in nonphotochemical quenching. FEBS Lett 582(25):3625-3631

Minagawa J (2011) State transitions-the molecular remodeling of photosynthetic supercomplexes that controls energy flow in the chloroplast. Biochim Biophys Acta 1807(8):897-905

Müller P, Li X, Niyogi KK (2001) Non-photochemical quenching. A response to excess light energy. Plant Physiol 125(4):1558

Müller MG, Lambrev P, Reus M, Wientjes E, Croce R, Holzwarth AR (2010) Singlet energy dissipation in the photosystem II lightharvesting complex does not involve energy transfer to carotenoids. Chemphyschem 11(6):1289-1296

Müller MG, Jahns P, Holzwarth AR (2013) Femtosecond transient absorption spectroscopy on the light-adaptation of living plants. EPJ Web Conf 41:08006

Murata N, Sugahara K (1969) Control of excitation transfer in photosynthesis. III. Light-induced decrease of chlorophyll a fluorescence related to photophosphorylation system in spinach chloroplasts. Biochim Biophys Acta 189(2):182-192

Nilkens M, Kress E, Lambrev P, Miloslavina Y, Mueller M, Holzwarth AR, Jahns P (2010) Identification of a slowly inducible zeaxanthin-dependent component of non-photochemical quenching of chlorophyll fluorescence generated under steady-state conditions in Arabidopsis. Biochim Biophys Acta 1797(4):466-475

Nishio JN, Whitmarsh J (1993) Dissipation of the proton electrochemical potential in intact chloroplasts (II. the $\mathrm{pH}$ gradient monitored by cytochrome $\mathrm{f}$ reduction kinetics). Plant Physiol 101(1):89-96 
Niyogi KK, Truong TB (2013) Evolution of flexible non-photochemical quenching mechanisms that regulate light harvesting in oxygenic photosynthesis. Curr Opin Plant Biol. 10.1016/j.pbi. 2013.03.011

Niyogi KK, Björkman O, Grossman AR (1997) The roles of specific xanthophylls in photoprotection. Proc Natl Acad Sci USA 94(25):14162-14167

Niyogi KK, Grossman AR, Björkman O (1998) Arabidopsis mutants define a central role for the xanthophyll cycle in the regulation of photosynthetic energy conversion. Plant Cell 10(7):1121-1134

Niyogi K, Shih C, Chow W, Pogson B, DellaPenna D, Bjorkman O (2001) Photoprotection in a zeaxanthin- and lutein-deficient double mutant of Arabidopsis. Photosynth Res 67(1-2):139-145

Niyogi KK, Li XP, Rosenberg V, Jung HS (2005) Is PsbS the site of non-photochemical quenching in photosynthesis. J Exp Bot 56(411):375-382

Noomnarm U, Clegg RM (2009) Fluorescence lifetimes: fundamentals and interpretations. Photosynth Res 101(2-3):181-194

Pascal AA, Liu ZZ, Broess KK, van Oort BB, van Amerongen HH, Wang CC, Horton PP, Robert BB, Chang WW, Ruban AA (2005) Molecular basis of photoprotection and control of photosynthetic light-harvesting. Nature 436(7047):134-137

Pogson BJ, Niyogi KK, Björkman O, DellaPenna D (1998) Altered xanthophyll compositions adversely affect chlorophyll accumulation and nonphotochemical quenching in Arabidopsis mutants. Proc Natl Acad Sci USA 95(22):13324-13329

Rees D, Noctor G, Ruban AV, Crofts J, Young A, Horton P (1992) pH dependent chlorophyll fluorescence quenching in spinach thylakoids from light treated or dark adapted leaves. Photosynth Res 31(1):11-19

Robert B (2009) Resonance Raman spectroscopy. Photosynth Res 101(2-3):147-155

Ruban AV, Walters RG, Horton P (1992) The molecular mechanism of the control of excitation energy dissipation in chloroplast membranes inhibition of $\mathrm{pH}$-dependent quenching of chlorophyll fluorescence by dicyclohexylcarbodiimide. FEBS Lett 309(2):175-179

Ruban AV, Berera R, Ilioaia C, van Stokkum IHM, Kennis JTM, Pascal AA, van Amerongen H, Robert B, Horton P, Grondelle RV (2007) Identification of a mechanism of photoprotective energy dissipation in higher plants. Nature 450(7169):575-578

Ruban AV, Johnson MP, Duffy CDP (2012) The photoprotective molecular switch in the photosystem II antenna. Biochim Biophys Acta 1817(1):167-181

Schneider AR, Geissler PL (2013) Coexistence between fluid and crystalline phases of proteins in photosynthetic membranes. Preprint arXiv/1302.6323v1 [cond-mat.soft]

Schreiber U (2004) Pulse-amplitude-modulation (PAM) fluorometry and saturation pulse method: an overview. Chlorophyll a luorescence: a signature of photosynthesis. Springer, Dordrect, The Netherlands, pp 279-319

Schreiber U, Schliwa U, Bilger W (1986) Continuous recording of photochemical and non-photochemical chlorophyll fluorescence quenching with a new type of modulation fluorometer. Photosynth Res 10(1):51-62

Schreiber U, Bilger W, Neubauer C (1994) Chlorophyll fluorescence as a nonintrusive indicator for rapid assessment of in vivo photosynthesis. In: Schulze ED, Caldwell MM (eds) Ecophysiology of photosynthesis. Springer, Dordrecht, pp 49-70

Schuldiner SS, Rottenberg HH, Avron MM (1972) Determination of $\mathrm{pH}$ in chloroplasts. 2. Fluorescent amines as a probe for the determination of $\mathrm{pH}$ in chloroplasts. FEBS J 25(1):64-70

Staehelin LA (2003) Chloroplast structure: from chlorophyll granules to supra-molecular architecture of thylakoid membranes. Photosynth Res 76(1-3):185-196

Takizawa K, Cruz JA, Kanazawa A, Kramer DM (2007) The thylakoid proton motive force in vivo. Quantitative, non-invasive probes, energetics, and regulatory consequences of light-induced pmf. Biochim Biophys Acta 1767(10):1233-1244

Terazono Y, Kodis G, Bhushan K, Zaks J, Madden C, Moore AL, Moore TA, Fleming GR, Gust D (2011) Mimicking the role of the antenna in photosynthetic photoprotection. J Am Chem Soc 133(9):2916-2922

Tian L, Farooq S, van Amerongen H (2013) Probing the picosecond kinetics of the photosystem II core complex in vivo. Phys Chem Chem Phys 15(9):3146

van Amerongen $\mathrm{H}$, van Grondelle R (2001) Understanding the energy transfer function of lhcII, the major light-harvesting complex of green plants. J Phys Chem B 105(3):604-617

van Amerongen H, Valkunas L, van Grondelle R (2000) Photosynthetic excitons. World Scientific Publishing Company Incorporated, Singapore City

van Oort B, Alberts M, de Bianchi S, Dall'Osto L, Bassi R, Trinkunas G, Croce R, van Amerongen H (2010) Effect of antennadepletion in photosystem II on excitation energy transfer in Arabidopsis thaliana. Biophys J 98(5):922-931

van Oort B, Maréchal A, Ruban AV, Robert B, Pascal AA, de Ruijter NCA, van Grondelle R, van Amerongen H (2011) Different crystal morphologies lead to slightly different conformations of lightharvesting complex II as monitored by variations of the intrinsic fluorescence lifetime. Phys Chem Chem Phys 13(27):12614

van Stokkum IHM, Larsen DS, van Grondelle R (2004) Global and target analysis of time-resolved spectra. Biochim Biophys Acta 1657(2-3):82-104

van Stokkum IHM, van Oort B, van Mourik F, Gobets B, van Amerongen H (2008) (Sub)-picosecond spectral evolution of fluorescence studied with a synchroscan streak-camera system and target analysis. Biophys Tech Photosynth 2:223-240

Walters RG, Ruban AV, Horton P (1996) Identification of protonactive residues in a higher plant light-harvesting complex. Proc Natl Acad Sci USA 93(24):14204-14209

Weiss JN (1997) The Hill equation revisited: uses and misuses. FASEB J 11(11):835-841

Wilk L, Grunwald M, Liao PN, Walla PJ, Kühlbrandt W (2013) Direct interaction of the major light-harvesting complex II and PsbS in nonphotochemical quenching. Proc Natl Acad Sci USA 110(14):5452-5456

van der Weij-de Wit CD, Dekker JP, van Grondelle R, van Stokkum IHM (2011) Charge separation is virtually irreversible in photosystem II core complexes with oxidized primary quinone acceptor. J Phys Chem A 115(16):3947-3956

Witt HT (1979) Energy conversion in the functional membrane of photosynthesis. Analysis by light pulse and electric pulse methods. The central role of the electric field. Biochim Biophys Acta 505(3-4):355-427

Wraight CA, Crofts AR (1970) Energy-dependent quenching of chlorophyll alpha fluorescence in isolated chloroplasts. Eur $\mathbf{J}$ Biochem 17(2):319-327

Yamamoto HY, Kamite L (1972) The effects of dithiothreitol on violaxanthin de-epoxidation and absorbance changes in the 500-nm region. Biochim Biophys Acta 267(3):538-543

Yang M, Damjanovic A, Vaswani HM, Fleming GR (2003) Energy transfer in photosystem I of cyanobacteria Synechococcus elongatus: model study with structure-based semi-empirical Hamiltonian and experimental spectral density. Biophys J 85(1):140-158

Zaks J Commented code for kinetic model of rapidly reversible nonphotochemical quenching. http://www.cchem.berkeley.edu/ grfgrp/jzaks/supp/html/index.html. Accessed 18 March 2013

Zaks J (2012) Regulatory dynamics of natural and artificial hotosynthesis. PhD Thesis, University of California, Berkeley

Zaks J, Amarnath K, Kramer DM, Niyogi KK, Fleming GR (2012) A kinetic model of rapidly reversible nonphotochemical quenching. Proc Natl Acad Sci USA 109(39):15757-15762 